\title{
Practical Hybrid Beamforming for Millimeter Wave Massive MIMO Full Duplex with Limited Dynamic Range
}

\author{
Chandan Kumar Sheemar, Student Member, IEEE, Christo Kurisummoottil Thomas, Member, IEEE, \\ and Dirk Slock, Fellow, IEEE
}

\begin{abstract}
Full-Duplex (FD) communication can revolutionize wireless communications as it doubles spectral efficiency and offers numerous other advantages over a half-duplex (HD) system. In this paper, we present a novel and practical joint hybrid beamforming (HYBF) and combining scheme for millimeter-wave (mmWave) massive MIMO FD system for weighted sum-rate (WSR) maximization with multi-antenna HD uplink and downlink users with non-ideal hardware. Moreover, we present a novel interference and self-interference (SI) aware optimal power allocation scheme for the optimal beamforming directions. The analog processing stage is assumed to be quantized, and both the unit-modulus and unconstrained cases are considered. Moreover, compared to the traditional sum-power constraints, the proposed algorithm is designed under the joint sum-power and the practical per-antenna power constraints. To model the non-ideal hardware of a hybrid FD transceiver, we extend the traditional limited dynamic range (LDR) noise model to mmWave. Our HYBF design relies on alternating optimization based on the minorization-maximization method. We investigate the maximum achievable gain of a hybrid FD system with different levels of the LDR noise variance and with different numbers of radio-frequency (RF) chains over a HD system. Simulation results show that the mmWave massive MIMO FD systems can significantly outperform the fully digital HD systems with only a few RF chains if the LDR noise generated from the limited number of RF chains available is low. If the LDR noise variance dominates, FD communication with HYBF results to be disadvantageous than a HD system.
\end{abstract}

Index Terms-Massive MIMO Full-Duplex, mmWave, Practical Hybrid Beamforming, Minorization-Maximization.

\section{INTRODUCTION}

$\mathbf{T}$ HE revolution in wireless communications has led to an exponential increase in the data rate requirements and users' number. Millimeter-wave (mmWave) frequency band $30-300 \mathrm{GHz}$ can accommodate the ever-increasing data demands and results to be a vital resource for future wireless communications [1]. It offers much wider bandwidths than the traditional cellular networks, and the available spectrum at such higher frequencies can be around 200 times greater [2]. Full-duplex (FD) communication in mmWave can enable simultaneous transmission and reception in the same frequency band, which theoretically doubles the spectral efficiency compared to half-duplex (HD) systems. By avoiding using two independent channels for bi-directional communication, it can be beneficial for efficient management of the available spectrum, reduce end-to-end delays/latency, solve the hidden node problem [3]-[5], and enable joint communication and sensing [6].

Self-interference (SI) is a key challenge to deal with to achieve an ideal FD operation, which can be $90-110$ $\mathrm{dB}$ higher than the received signal power [7], [8]. Given the tremendous amount of SI, signal reception is impossible without a proper SI cancellation (SIC) scheme. Beamforming serves as a potential tool to mitigate SI while jointly serving multiple users [9]-[18], and can lead us closer to two times improvement in the spectral efficiency compared to HD systems. However, in practice, the maximum achievable gain of the beamforming schemes is limited by the limited dynamic range (LDR) of the radio frequency (RF) circuitry. Components like

Chandan Kumar Sheemar and Dirk Slock are with the communication systems department at EURECOM, Sophia Antipolis, 06410, France (emails:sheemar@eurecom.fr,slock@eurecom.fr);

Christo Kurisummoottil Thomas is with Qualcomm Finland RFFE Oy, Keilaranta 8, 02150 Espoo (e-mail: ckurisum@qti.qualcomm.com). power amplifiers (PAs), analog-to-digital-converters (ADCs), mixers and low noise power amplifiers (LNA) add additional LDR noise, which increases the overall noise floor at the FD node. Therefore, for correct performance characterization of the beamforming designs, the undesirable LDR effect of the RF circuitry must be considered with the LDR noise model [10]-[18], which makes them impairments aware.

Digital beamforming designs for FD under the LDR noise model helped to understand the impact of the non-ideal hardware and how it directly impacts the maximum achievable performance at lower frequencies. [10]-[18]. However, shifting the potential of FD to the mmWave is much more challenging. The signal can suffer from shadowing effects, higher Doppler spreads, rapid channel fluctuations, and intermittent connectivity [2], [19]. Due to these propagation challenges, the total received signal at the FD node can manifest a very low signal-to-self-interference-plus-noise-ratio (SSINR), given that the useful signal undergoes considerably a larger path-loss than the SI signal. To overcome the propagation challenges, FD base stations must be designed with a massive number of antennas. But, enabling beamforming to manage interference and SI in mmWave FD systems with a massive number of antenna elements based only on digital processing [10]-[18] would require hundreds of RF chains, which is prohibitive due to hardware cost.

\section{A. State-of-the-art on mmWave FD}

The mmWave massive MIMO FD systems can be built costefficiently only with fewer radio-frequency (RF) chains than the number of antennas. Thus, they have to rely on efficient hybrid beamforming (HYBF) designs [19]-[30], consisting of large-dimensional phasor processing in the analog domain and lower-dimensional digital processing. HYBF is beneficial to 
mitigate the SI before the total received signal presents at the input of the ADCs. Thus, improving the SSINR and preserving more of their dynamic range to capture the useful received signal of interest.

In [20], the authors proposed the first HYBF design for an amplify and forward FD relay for the mmWave backhaul link. In [21], a single stream HYBF for two bidirectional MIMO FD nodes is studied. In [22], HYBF for an FD relay assisted mmWave macro-cell scenario is investigated. In [23], HYBF for two mmWave MIMO bidirectional FD nodes is presented. In [31], HYBF for FD integrated access and backhaul is presented. In [19], the authors proposed joint HYBF and combining for a point-to-point FD communication. In [24], a novel HYBF design for an FD mmWave MIMO relay is proposed. In [25], a machine-learning based HYBF for a oneway mmWave FD relay is investigated. In [26], HYBF for a bidirectional point-to-point OFDM FD system is available. In [27], the authors propose a modified zero-forcing maxpower design with HYBF for two massive MIMO FD nodes. In [28], a joint HYBF and combining with optimal power control for the uplink users in a multi-cell mmWave FD system with single-antenna users is presented. In [29], a low-cost phasor design for HYBF in a single-cell mmWave MIMO FD system with single-antenna users is proposed. In [30], a novel HYBF design with one uplink and one downlink multi-antenna HD user only under the receive side LDR is proposed. In [32], HYBF and combining design for two massive MIMO nodes, that simultaneously maximize the sum spectral efficiency and cancel the SI in the analog domain by keeping the signal level at the input of the ADCs under control is proposed. In [33], HYBF for two fully connected nodes that approaches SI-free sum-spectral efficiency is proposed. In [34], HYBF for mmWave equipped with analog beamforming stage is presented. In [35], hybrid beamforming for point-topoint mmWave FD massive MIMO interference channel is presented. In [36], frequency-selective HYBF for a wide-band mmWave FD systems is proposed.

The state-of-the-art contains novel HYBF designs which are limited to multiple single-antenna uplink and downlink users communicating simultaneously, multi-antenna two massive MIMO FD nodes communicating with each other, or FD relays serving downlink users. The only contribution similar to the work considered in this paper is available in [30]. The design is limited to one uplink and one downlink user, and it is motivated from the receive side LDR to avoid saturation of the ADCs, which result to be the most dominant noise contribution for FD. However, also the transmit side LDR, mainly due to non-ideal power amplifiers (PAs), contributes significantly [37]. In [30], also the cross interference generated from the uplink user towards the downlink user is ignored, which can have a major impact on the maximum achievable performance. The effect of cross-interference generated from opposite transmission directions in different cells is wellinvestigated in dynamic time division duplexing (TDD) [38][42]. Its effect in FD communication is even much more severe as it occurs in the same cell. In particular, in small cells, whose deployment is expected in the future cellular networks [43], the base station and the users operate with similar transmission power [42]. For the case of one uplink and one downlink user as in [30] in a small cell, if the users are operating far from the base station and very close the each other, then the crossinterference contribution can become as severe as the SI. In the FD multi-user case, with multiple uplink users operating near the downlink users and far from the base station, each downlink user sees the cross-interference, which is summed over the transmission power of all the uplink users (with each user transmitting with similar power as the base station). In such a case, the cross-interference can become even more severe than the SI, if not considered in the beamforming designs. Therefore, cross-interference is an important aspect, as the SI, for the deployment of FD systems for multi-user communication. The SI at the FD node aims to improve the uplink rate, and cross-interference management is required to improve the downlink rate. Only by properly mitigating both we can get closer to the two times improvement in the spectral efficiency expected from FD multi-user systems.

Traditionally, the analog domain processing consisted only of the unit amplitude phase-shifters. However, recently new transceivers have started to emerge which also allow amplitude manipulation (denoted as amplitude tapering in the antenna theory literature) in the analog domain for the base station consisting of large number of antenna, with the aid of amplitude modulators (AMs) [44], [45]. The only reference on FD communication to consider amplitude control for $\mathrm{HYBF}$ design is [30].

\section{B. Main Contributions}

This paper presents a novel HYBF and combining design for weighted-sum-rate (WSR) maximization problem in a single cell with multiple multi-antenna HD uplink, and downlink users served simultaneously with multiple streams by a massive MIMO FD base station. We assume that all the communication nodes are equipped with non-ideal hardware. For the uplink and downlink users, the non-ideal hardware is modelled with the traditional LDR noise model [29], and for the FD base-station, we extend the LDR model from [29] to the case of a hybrid transceiver. The WSR maximization problem is formulated in terms of digital beamformers, ana$\log$ beamformer and combiner, and solved with alternating optimization based on the minorization-maximization method [46]. Finally, we propose a novel SI, impairments (LDR) and cross-interference aware optimal power allocation scheme to include the optimal power allocation for the uplink users and the base station.

The proposed design also considers the joint practical perantenna power constraints and the sum-power constraints. The sum-power constraints at each terminal are imposed by the regulations, which limit the total transmit power. In practice, each transmit antenna is equipped with its PA [47] and the perantenna power constraints arise due to the power consumption limits imposed on the physical PAs [47]-[50]. We remark that also the massive MIMO systems are expected to be deployed with one PA per antenna, as this enables the deployment of very low cost PAs with output power of the order of milli-Watt [51]. The joint sum-power and per-antenna power 


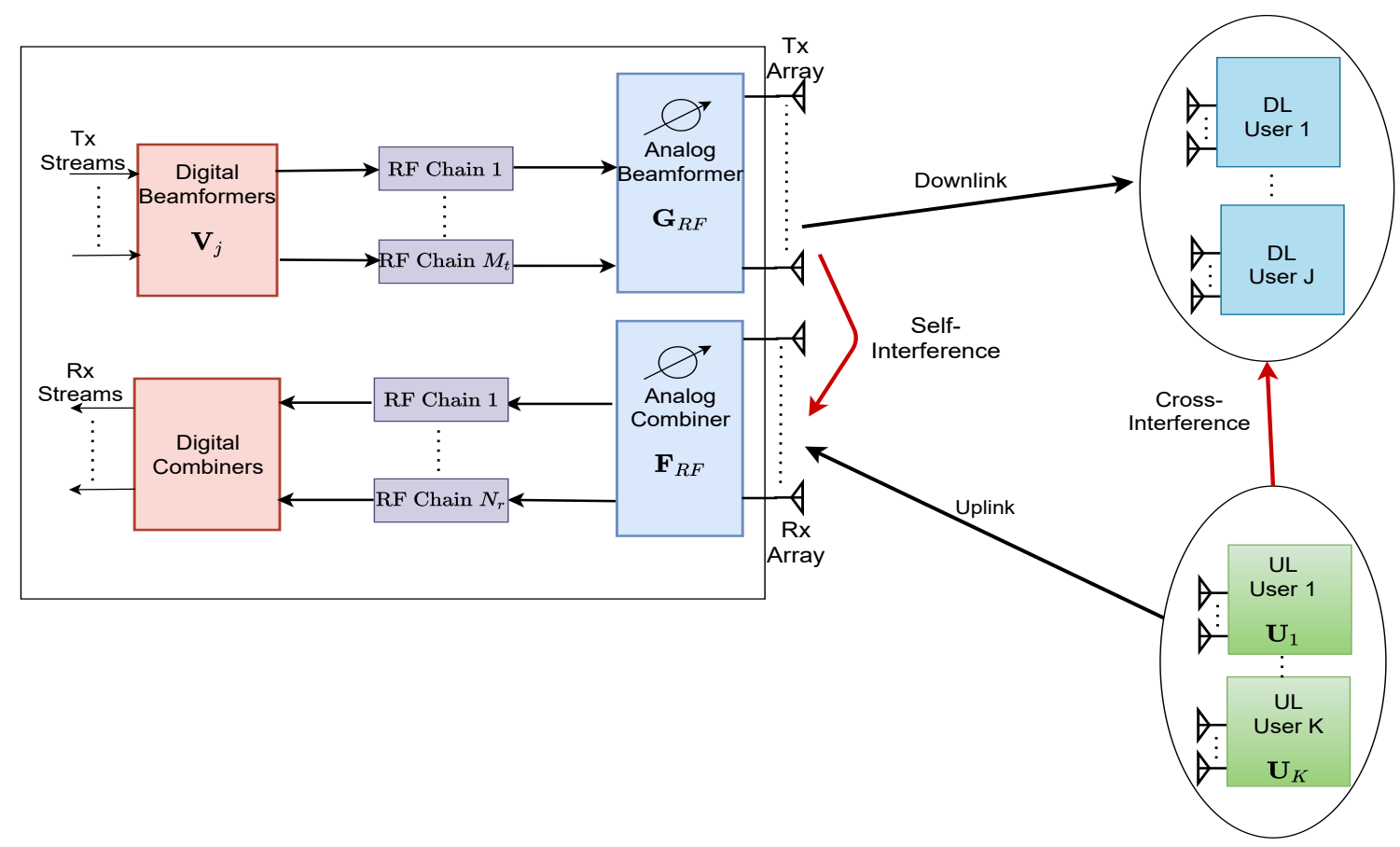

Fig. 1: Full-Duplex communication in mmWave band with hybrid beamforming and combining. Tx, Rx, UL and DL denote transmit, receive, uplink and downlink, respectively.

constraints consider both the regulations and the physical limits of the hardware to optimize the communication system's performance. In practice, the analog beamforming and combining stage must satisfy the unit-modulus constraint, and the phase-shifters can only assume discrete values. If AMs are available, the unit-modulus constraint is relaxed, but they add up additional hardware costs. Therefore, in this work, the analog stage is distinguished in unit-modulus and quantized phase-shifters or unconstrained with quantized phases and amplitudes.

Our simulation results show significant performance improvement for a hybrid FD system over a fully digital HD system with only a few RF chains. We also show that different levels of the LDR noise variance lead to different levels of achievable gain. Moreover, we show that AMs with a few RF chains can improve the performance of HYBF schemes considerably, if they are affected by a moderate to low LDR noise.

In summary, the contributions of our work are:

- Introduction of the WSR maximization problem for HYBF and combining in mmWave massive MIMO FD system with multi-antenna HD uplink and downlink users served with multiple streams.

- Motivated by practical considerations, the LDR noise model for the non-ideal hardware and the per-antenna power constraints to represent the physical limits of the PAs, both at the base station and the uplink users are also considered.

- A novel SI, LDR/impairments and cross-interference aware optimal power allocation scheme for multi-antenna uplink users and the mmaWave hybrid FD base station is proposed.
The rest of the paper is organized as follows. In Section II we present the system model and introduce the problem formulation for WSR maximization under the joint constraints and extend the LDR noise model to mmWave. Section III introduces the minorization-maximization method to leverage alternating optimization. Section IV presents the novel HYBF and combining design. Finally, Section V and VI present the simulation results and conclusions, respectively.

Notations: Boldface lower and upper case characters denote vectors and matrices, respectively, $E\{\cdot\}, \operatorname{Tr}\{\cdot\},(\cdot)^{H},(\cdot)^{T}$, $\otimes, \boldsymbol{I}$, and $\boldsymbol{D}_{d}$ denote expectation, trace, conjugate transpose, transpose, kronecker product, identity matrix and the $d$ dominant vector selection matrix, respectively. The operator $\operatorname{vec}(\boldsymbol{X})$ stacks the column of $\boldsymbol{X}$ into a vector $\boldsymbol{x}$, unvec $(\boldsymbol{x})$ reshape $\boldsymbol{x}$ into $\boldsymbol{X}$ and $\angle \boldsymbol{X}$ returns the matrix $\boldsymbol{X}$ with unitmodulus amplitudes (so only the phase part). The operators $\operatorname{Cov}(\cdot)$, and $\operatorname{diag}(\cdot)$ denote the covariance and diagonal matrices, respectively, and $\operatorname{SVD}(\boldsymbol{X})$ returns the singular value decomposition of $\boldsymbol{X}$. Elements of matrix $\boldsymbol{X}$ at $m$-th row and $n$-th column is denoted with $\boldsymbol{X}(m, n)$.

\section{SySTEM MOdEL}

We consider a single-cell mmWave massive MIMO FD communication system consisting of one massive MIMO FD base station equipped with $M_{t}$ transmit and $N_{r}$ receive RF chains, $M_{0}$ transmit and $N_{0}$ receive antennas, respectively, serving simultaneously $J$ downlink and $K$ uplink users, as shown in Fig. 1. We assume perfect channel state information $(\mathrm{CSI})^{1}$. Let $\mathcal{U}=\{1, \ldots, K\}$ and $\mathcal{D}=\{1, \ldots, J\}$ contain the

\footnotetext{
${ }^{1}$ The CSI of mmWave FD systems can be acquired based on compressed sensing channel estimation techniques discussed in [52] for mmWave HD system and it is part of the ongoing research [53].
} 
indices of the served multi-antenna HD uplink and downlink users, respectively. Let $M_{k}$ and $N_{j}$ denote the number of transmit and receive antennas at the $k$-th uplink and $j$ th downlink user, respectively. We consider a multi-stream approach and the number of data streams transmitted from $k$-th uplink and to $j$-th downlink user are denoted with $u_{k}$ and $v_{j}$, respectively. Let $\boldsymbol{U}_{k} \in \mathbb{C}^{M_{k} \times u_{k}}$ and $\boldsymbol{V}_{j} \in \mathbb{C}^{M_{t} \times v_{j}}$ denote the precoders for the white, unitary variance data streams $\boldsymbol{v}_{k} \in \mathbb{C}^{u_{k} \times 1}$ and $\boldsymbol{v}_{j} \in \mathbb{C}^{v_{j} \times 1}$, respectively. Let $\boldsymbol{G}_{R F} \in \mathbb{C}^{M_{0} \times M_{t}}$ and $\boldsymbol{F}_{R F} \in \mathbb{C}^{N_{r} \times N_{0}}$ denote the fully connected analog beamformer and combiner at the FD base station, respectively. As the analog stage assumes only discrete values, let $\mathcal{P}=\left\{a_{0}, a_{1} \omega, a_{2} \omega^{2}, \ldots, a_{n_{p s}-1} \omega^{n_{p s}-1}\right\}$, denote the set of $n_{p_{s}}$ complex values with the phase part $\omega=e^{\frac{j 2 \pi}{n_{p s}}}$ and amplitudes $a_{i}$, with $0 \leq i \leq n_{p s-1}$. In the case of unit-modulus phase-shifters, the values of amplitudes $a_{i}$ results to be $1 \forall i$. We define the quantizer function $\mathbb{Q}(\cdot)$ which quantizes the phase at the $m$-th row and $n$-th column $\boldsymbol{G}_{R F}(m, n)\left(\right.$ or $\boldsymbol{F}_{R F}(m, n)$ ) such that $\mathbb{Q}\left(\boldsymbol{G}_{R F}(m, n)\right) \in \mathcal{P}$ (or $\mathbb{Q}\left(\boldsymbol{F}_{R F}(m, n)\right) \in \mathcal{P}$ ). The thermal noise vectors at the FD base station and at the $j$-th downlink user is dentoed with $\boldsymbol{n}_{0} \sim \mathcal{C N}\left(0, \sigma_{0}^{2} \boldsymbol{I}_{N_{0}}\right)$ and $\boldsymbol{n}_{j} \sim \mathcal{C N}\left(0, \sigma_{j}^{2} \boldsymbol{I}_{N_{j}}\right)$ denote , respectively. The transmitter and receiver distortions at the $k$-th uplink and $j$-th downlink user (LDR model) are denoted with $\boldsymbol{c}_{\boldsymbol{k}}$ and $\boldsymbol{e}_{j}$, respectively, and be modelled as [12]

$$
\begin{gathered}
\boldsymbol{c}_{k} \sim \mathcal{C N}\left(\boldsymbol{0}_{M_{k} \times 1}, k_{k} \operatorname{diag}\left(\boldsymbol{U}_{k} \boldsymbol{U}_{k}^{H}\right)\right), \\
\boldsymbol{e}_{\boldsymbol{j}} \sim \mathcal{C N}\left(\mathbf{0}_{N_{j} \times 1}, \beta_{j} \operatorname{diag}\left(\boldsymbol{\Phi}_{j}\right)\right),
\end{gathered}
$$

where $k_{k} \ll 1, \beta_{j} \ll 1$ and $\boldsymbol{\Phi}_{j}=\operatorname{Cov}\left(\boldsymbol{r}_{j}\right)$, where $\boldsymbol{r}_{j}$ denotes the undistorted received vector. The transmitter and receiver distortions at the FD base station are denoted with $c_{0}$ and $e_{0}$, respectively, and modelled as

$$
\begin{gathered}
\boldsymbol{c}_{0} \sim \mathcal{C N}\left(\mathbf{0}_{M_{0} \times 1}, k_{0} \operatorname{diag}\left(\sum_{n \in \mathcal{D}} \boldsymbol{G}_{R F} \boldsymbol{V}_{n} \boldsymbol{V}_{n}^{H} \boldsymbol{G}_{R F}^{H}\right)\right), \\
\boldsymbol{e}_{\mathbf{0}} \sim \mathcal{C} \mathcal{N}\left(\mathbf{0}_{N_{0} \times 1}, \beta_{0} \operatorname{diag}\left(\boldsymbol{\Phi}_{0}\right)\right)
\end{gathered}
$$

where $k_{0} \ll 1, \beta_{0} \ll 1, \Phi_{0}=\operatorname{Cov}\left(\boldsymbol{r}_{0}\right)$, where $\boldsymbol{r}_{0}=\boldsymbol{y}-\boldsymbol{e}_{0}$ is the undistorted received signal, where $\boldsymbol{y}$ is given in (5). Note that (3) extends the transmit LDR noise model [12] to the case of hybrid FD transceiver at the transmit side. For the receive side, the ADCs are placed after the analog combiner which are a major source (the most dominant) of LDR noise, therefore to account for that, $\boldsymbol{e}_{0}$ must be injected after $\boldsymbol{F}_{R F}$ (Please see (5)). Moreover, we remark that (3)-(4) is a simplified LDR noise model. Even though in the massive MIMO transceivers, each antenna has its own PA [51] (the most dominant source of LDR noise at the transmit side [37]), some of the circuitry is shared among multiple antennas. Thus, it can lead to some correlation among the distortions, which is different for the fully-connected and the partially-connected hybrid transceivers and must be investigated with experimental measurements.
The signal received at the FD base station after the analog combiner and at the $j$-th downlink user, can be written as

$$
\begin{gathered}
\boldsymbol{y}=\boldsymbol{F}_{R F} \sum_{k \in \mathcal{U}} \boldsymbol{H}_{k} \boldsymbol{U}_{k} \boldsymbol{s}_{k}+\boldsymbol{F}_{R F} \sum_{k \in \mathcal{U}} \boldsymbol{H}_{k} \boldsymbol{U}_{k} \boldsymbol{c}_{k}+\boldsymbol{F}_{R F} \boldsymbol{n}_{0} \\
+\boldsymbol{F}_{R F} \boldsymbol{H}_{0} \sum_{j \in \mathcal{D}} \boldsymbol{G}_{R F} \boldsymbol{V}_{j} \boldsymbol{s}_{j}+\boldsymbol{F}_{R F} \boldsymbol{H}_{0} \boldsymbol{c}_{0}+\boldsymbol{e}_{0}, \\
\boldsymbol{y}_{j}=\boldsymbol{H}_{j} \sum_{n \in \mathcal{D}} \boldsymbol{G}_{R F} \boldsymbol{V}_{n} \boldsymbol{s}_{n}+\boldsymbol{H}_{j} \sum_{n \in \mathcal{D}} \boldsymbol{G}_{R F} \boldsymbol{V}_{n} \boldsymbol{c}_{0}+\boldsymbol{e}_{j}+\boldsymbol{n}_{j} \\
+\sum_{k \in \mathcal{U}} \boldsymbol{H}_{j, k} \boldsymbol{U}_{k} \boldsymbol{s}_{k}+\sum_{k \in \mathcal{U}} \boldsymbol{H}_{j, k} \boldsymbol{c}_{k} .
\end{gathered}
$$

The matrices $\boldsymbol{H}_{k} \in \mathbb{C}^{N_{0} \times M_{k}}$ and $\boldsymbol{H}_{j} \in \mathbb{C}^{N_{j} \times M_{0}}$ denote the $k$-th UL and $j$-th DL user channel, respectively, $\boldsymbol{H}_{0} \in$ $\mathbb{C}^{N_{0} \times M_{0}}$ and $\boldsymbol{H}_{j, k} \in \mathbb{C}^{N_{j} \times M_{k}}$ denote the self-interference and the cross-interference channel from $k$-th uplink to the $j$ th downlink user, respectively. At the mmWave, the uplink users channel can be modelled as [19]

$$
\boldsymbol{H}_{k}=\sqrt{\frac{M_{k} N_{0}}{N_{c} N_{p}}} \sum_{n_{c}=1}^{N_{c}} \sum_{n_{p}=1}^{N_{p}} \alpha_{k}^{\left(n_{p}, n_{c}\right)} \boldsymbol{a}_{r}\left(\phi_{k}^{n_{p}, n_{c}}\right) \boldsymbol{a}_{t}^{T}\left(\theta_{k}^{n_{p}, n_{c}}\right),
$$

where $N_{c}$ and $N_{p}$ denote the number of clusters and number of rays, respectively, $\alpha_{k}^{\left(n_{p}, n_{c}\right)} \sim \mathcal{C N}(0,1)$ is a complex Gaussian random variable with amplitudes and phases distributed according to the Rayleigh and uniform distribution, respectively, and $\boldsymbol{a}_{r}\left(\phi_{k}^{n_{p}, n_{c}}\right)$ and $\boldsymbol{a}_{t}^{T}\left(\theta_{k}^{n_{p}, n_{c}}\right)$ denote the receive and transmit antenna array response with angle of arrival (AoA) $\phi_{k}^{n_{p}, n_{c}}$ and angle of departure (AoD) $\theta_{k}^{n_{p}, n_{c}}$, respectively. The downlink channels $\boldsymbol{H}_{j}$ and the cross-interference channels $\boldsymbol{H}_{j, k}$ can be modelled similarly as in (7). The SI channel can be modelled as [19]

$$
\boldsymbol{H}_{0}=\sqrt{\frac{\kappa}{\kappa+1}} \boldsymbol{H}_{L o S}+\sqrt{\frac{1}{\kappa+1}} \boldsymbol{H}_{r e f},
$$

with the Rician factor $\kappa$, and the line-of-sight $(\operatorname{LoS})$ and reflected contributions of the SI signal denoted with $\boldsymbol{H}_{L o S}$ and $\boldsymbol{H}_{r e f}$, respectively. The channel matrix $\boldsymbol{H}_{r e f}$ can be modelled as in (7) and the element in the $m$-th row and $n$-th columns of $\boldsymbol{H}_{L o S}$ can be modelled as [19]

$$
\boldsymbol{H}_{L o S}(m, n)=\frac{\rho}{r_{m, n}} e^{-j 2 \pi \frac{r_{m, n}}{\lambda}}
$$

where $\rho$ is the power normalization constant which assure that $\mathbb{E}\left(\left\|\boldsymbol{H}_{L o S}(m, n)\right\|_{F}^{2}\right)=M_{0} N_{0}, r_{m, n}$ is the distance parameter and can be modelled as in (9) [19], in the case of uniform linear arrays (ULAs). Table I summarizes all the notations.

Let $\bar{k}$ and $\bar{j}$ denote the indices in the set $\mathcal{U}$ and $\mathcal{D}$, without the element $k$ and $j$, respectively. The received (signal plus) interference and noise covariance matrices from user $k \in \mathcal{U}$ and at the user $j \in \mathcal{D}$ are denoted with $\left(\boldsymbol{R}_{k}\right) \boldsymbol{R}_{\bar{k}}$ and $\left(\boldsymbol{R}_{j}\right)$ $\boldsymbol{R}_{\bar{j}}$, respectively. Let $\boldsymbol{T}_{k}, \forall k \in \mathcal{U}$, and $\boldsymbol{Q}_{j}, \forall j \in \mathcal{D}$, defined as

$$
\boldsymbol{T}_{k}=\boldsymbol{U}_{k} \boldsymbol{U}_{k}^{H}, \quad \boldsymbol{Q}_{j}=\boldsymbol{G}_{R F} \boldsymbol{V}_{j} \boldsymbol{V}_{j}^{H} \boldsymbol{G}_{R F}^{H},
$$

be the transmitted covariance matrices from user $k \in \mathcal{U}$ and for user $j \in \mathcal{D}$. By considering the effect of the non-ideal hardware, cross-interference, interference and self-interference, the 


$$
\begin{gathered}
\boldsymbol{R}_{k}=\underbrace{\boldsymbol{F}_{R F} \boldsymbol{H}_{k} \boldsymbol{T}_{k} \boldsymbol{H}_{k}^{H} \boldsymbol{F}_{R F}^{H}}_{\triangleq \boldsymbol{S}_{k}}+\sum_{\substack{i \in \mathcal{U} \\
i \neq k}} \boldsymbol{F}_{R F} \boldsymbol{H}_{i} \boldsymbol{T}_{i} \boldsymbol{H}_{i}^{H} \boldsymbol{F}_{R F}^{H}+\sum_{i \in \mathcal{U}} k_{i} \boldsymbol{F}_{R F} \boldsymbol{H}_{i} \operatorname{diag}\left(\boldsymbol{T}_{i}\right) \boldsymbol{H}_{i}^{H} \boldsymbol{F}_{R F}^{H}+\sigma_{0}^{2} \boldsymbol{I}_{N_{0}} \\
+\boldsymbol{F}_{R F} \boldsymbol{H}_{0}\left(\sum_{n \in \mathcal{D}} \boldsymbol{Q}_{n}+k_{0} \operatorname{diag}\left(\sum_{n \in \mathcal{D}} \boldsymbol{Q}_{n}\right)\right) \boldsymbol{H}_{0}^{H} \boldsymbol{F}_{R F}^{H}+\beta_{0} \operatorname{diag}\left(\boldsymbol{\Phi}_{0}\right), \\
\boldsymbol{R}_{j} \underbrace{\boldsymbol{H}_{j} \boldsymbol{Q}_{j} \boldsymbol{H}_{j}^{H}}_{\triangleq \boldsymbol{S}_{j}}+\boldsymbol{H}_{j} \sum_{\substack{n \in \mathcal{D} \\
n \neq j}} \boldsymbol{Q}_{n} \boldsymbol{H}_{j}^{H}+k_{0} \boldsymbol{H}_{j} \operatorname{diag}\left(\sum_{n \in \mathcal{D}} \boldsymbol{Q}_{n}\right) \boldsymbol{H}_{j}^{H}+\sigma_{j}^{2} \boldsymbol{I}_{N_{j}}+\sum_{i \in \mathcal{U}} \boldsymbol{H}_{j, i}\left(\boldsymbol{T}_{i}+k_{i} \operatorname{diag}\left(\boldsymbol{T}_{i}\right)\right) \boldsymbol{H}_{j, i}^{H}+\beta_{j} \operatorname{diag}\left(\boldsymbol{\Phi}_{j}\right), \\
\boldsymbol{R}_{\bar{k}}=\boldsymbol{R}_{k}-\boldsymbol{S}_{k}, \quad \boldsymbol{R}_{j}=\boldsymbol{R}_{j}-\boldsymbol{S}_{j} .
\end{gathered}
$$

\section{TABLE I: Notations}

\begin{tabular}{|l|l|}
\hline$M_{t}$ & Transmit RF chains for the FD base station \\
\hline$N_{r}$ & Receive RF chains for the FD base station \\
\hline$M_{0}$ & Transmit antennas at the FD base station \\
\hline$N_{0}$ & Receive antennas at the FD base station \\
\hline$M_{k}$ & Transmit antennas for the $k$-th uplink user \\
\hline$N_{j}$ & Receive antennas for the $j$-th downlink user \\
\hline $\boldsymbol{U}_{k}$ & Digital beamformer for $k$-th uplink user \\
\hline $\boldsymbol{V}_{j}$ & Digital beamformer for the $j$-th downlink user \\
\hline $\boldsymbol{G}_{R F}$ & Analog beamformer at the FD base station \\
\hline $\boldsymbol{F}_{R F}$ & Analog combiner at the FD base station \\
\hline $\boldsymbol{c}_{k}$ & Transmit LDR noise from the $k$-th uplink user \\
\hline $\boldsymbol{c}_{0}$ & Transmit LDR noise from the FD base station \\
\hline $\boldsymbol{e}_{0}$ & Receive LDR noise at the FD base station \\
\hline $\boldsymbol{e}_{j}$ & Receive LDR noise at the $j$-th downlink user \\
\hline $\boldsymbol{n}_{0}$ & Thermal noise at the FD base station \\
\hline $\boldsymbol{n}_{j}$ & Thermal noise at the downlink user $j$ \\
\hline $\boldsymbol{H}_{0}$ & SI channel \\
\hline $\boldsymbol{H}_{k}$ & Direct channel for the $k$-th uplink user \\
\hline $\boldsymbol{H}_{j}$ & Direct channel for the $j$-th uplink user \\
\hline $\boldsymbol{H}_{j, k}$ & $\begin{array}{l}\text { cross-interference channel between uplink } \\
\text { user } k \text { and downlink user } j \text {. }\end{array}$ \\
\hline
\end{tabular}

received covariance matrices at the base station after the analog combiner $\left(\boldsymbol{R}_{k}\right.$ and $\left.\boldsymbol{R}_{\bar{k}}\right)$, and at the downlink user $j \in \mathcal{D}$ $\left(\boldsymbol{R}_{j}\right.$ and $\boldsymbol{R}_{\bar{j}}$ ) can be written as in (11), shown at the top of the next page, where $\boldsymbol{S}_{k}$ and $\boldsymbol{S}_{j}$ denote the useful signal part transmitted from user $k \in \mathcal{U}$ and for user $j \in \mathcal{D}$, respectively. The undistorted received covariance matrices can be recovered from (11) as $\boldsymbol{\Phi}_{0}=\boldsymbol{R}_{k}\left(\beta_{0}=0\right)$ and $\boldsymbol{\Phi}_{j}=\boldsymbol{R}_{j}\left(\beta_{j}=0\right)$.

The WSR maximization problem with respect to HYBF and combining together with the uplink beamformers for a single cell mmWave massive MIMO FD system, serving simultaneously $J$ downlink and $K$ uplink multi-antenna users, under the joint sum-power and per-antenna constraints, and discrete analog processing stage can be stated as

$$
\max _{\substack{U, \boldsymbol{V}, \boldsymbol{G}_{R F} \boldsymbol{F}_{R F}}} \sum_{k \in \mathcal{U}} w_{k} \ln \operatorname{det}\left(\boldsymbol{R}_{\bar{k}}^{-1} \boldsymbol{R}_{k}\right)+\sum_{j \in \mathcal{D}} w_{j} \ln \operatorname{det}\left(\boldsymbol{R}_{\bar{j}}^{-1} \boldsymbol{R}_{j}\right)
$$

$$
\begin{array}{ll}
\text { s.t. } & \operatorname{diag}\left(\boldsymbol{U}_{k} \boldsymbol{U}_{k}^{H}\right) \preceq \boldsymbol{\Lambda}_{\boldsymbol{k}}, \quad \forall k \in \mathcal{U}, \\
& \operatorname{diag}\left(\sum_{j \in \mathcal{D}} \boldsymbol{G}_{R F} \boldsymbol{V}_{j} \boldsymbol{V}_{j}^{H} \boldsymbol{G}_{R F}^{H}\right) \preceq \boldsymbol{\Lambda}_{0},
\end{array}
$$

$$
\begin{gathered}
\operatorname{Tr}\left(\boldsymbol{U}_{k} \boldsymbol{U}_{k}^{H}\right) \preceq \alpha_{k}, \quad \forall k \in \mathcal{U}, \\
\operatorname{Tr}\left(\sum_{j \in \mathcal{D}} \boldsymbol{G}_{R F} \boldsymbol{V}_{j} \boldsymbol{V}_{j}^{H} \boldsymbol{G}_{R F}^{H}\right) \preceq \alpha_{0} . \\
\boldsymbol{G}_{R F}(m, n) \in \mathcal{P}, \quad \forall m, n, \\
\boldsymbol{F}_{R F}(i, j) \in \mathcal{P}, \quad \forall i, j .
\end{gathered}
$$

The scalars $w_{k}$ and $w_{j}$ denote the uplink and downlink rate weights, $\boldsymbol{\Lambda}_{\boldsymbol{k}}$ and $\boldsymbol{\Lambda}_{\mathbf{0}}$ are the $M_{k} \times M_{k}$ and $M_{0} \times M_{0}$ diagonal per-antenna power matrices for user $k \in \mathcal{U}$ and the FD base station, respectively, $\alpha_{k}$ and $\alpha_{0}$ denote their total sum-power constraints, respectively, $\boldsymbol{U}$ and $\boldsymbol{V}$ denote the collection of digital uplink and downlink beamformers and combiners, respectively. The constraints (12f)-(12g) denote the discretization constraints on the phase and the amplitude part for the analog beamformer and combiner, respectively.

Remark: Note that by stating the rate maximization problem (12) as $\log \operatorname{det}(\cdot)$, it not affected by the digital receivers. It assumes the well-known optimal minimum mean-squared error (MMSE) digital receivers on the receive side (of the size of the number of antennas). The rate achieved with (12) is the same after the MMSE receivers (For more details, see (4) - (9) [54]). The FD base station has analog combiner $\boldsymbol{F}_{R F}$, which has a special structure (common to all uplink users), and it reduces the dimensions of the covariance matrices, hence the rate is affected. So, for WSR maximization purpose, from the receiver point of view, only the analog combiner of the base station must be considered in the design.

\section{Minorization-MAXimization}

The problem (12) under the joint per-antenna and sumpower constraints is non-concave in $\boldsymbol{T}_{k}$ and $\boldsymbol{Q}_{j}$, due to interference, which leads to finding the global optimum solution very challenging. In this section, we adopt the minorizationmaximization method [46], which enables to leverage alternating optimization to update all the variables iteratively and thus solve (12) to a local optima. It consists in updating one variable, while keeping the remaining one fixed and the complete information about the fixed variables is summarized in the gradients, which are updated at each iteration.

The WSR problem (12) is reformulated at each iteration with a concave reformulation with its minorizer, using the 
difference-of-convex (DC) programming [55] in terms of the variable to be updated, while the other variables are fixed. The WSR in (12) can be written with weighted-rate (WR) of user $k \in \mathcal{U}\left(\mathrm{WR}_{k}^{U L}\right)$, user $j \in \mathcal{D}\left(\mathrm{WR}_{j}^{D L}\right)$, WSR for $\bar{k}\left(\mathrm{WSR}_{k}^{U}{ }^{L}\right)$ and $\bar{j}\left(\mathrm{WSR}_{\bar{j}}^{D L}\right)$ as

$$
\mathrm{WSR}=\underbrace{\mathrm{WR}_{k}^{U L}+\mathrm{WSR}_{k}^{U L}}_{\triangleq \mathrm{WSR}^{U L}}+\underbrace{\mathrm{WR}_{j}^{D L}+\mathrm{WSR}_{\bar{j}}^{D L}}_{\triangleq \mathrm{WSR}^{D L}},
$$

where $\mathrm{WSR}^{U L}$ and $\mathrm{WSR}^{D L}$ denote the WSR in uplink and downlink, respectively, and

$$
\begin{gathered}
\mathrm{WR}_{k}^{U L}=w_{k} \ln \operatorname{det}\left(\boldsymbol{R}_{\bar{k}}^{-1} \boldsymbol{R}_{k}\right), \\
\mathrm{WSR}_{\bar{k}}^{L}=\sum_{i \in \mathcal{U}, i \neq k} w_{i} \ln \operatorname{det}\left(\boldsymbol{R}_{\bar{i}}^{-1} \boldsymbol{R}_{i}\right), \\
\mathrm{WR}_{j}^{D L}=w_{j} \ln \operatorname{det}\left(\boldsymbol{R}_{\bar{j}}^{-1} \boldsymbol{R}_{j}\right), \\
\mathrm{WSR}_{\bar{j}}^{D L}=\sum_{n \in \mathcal{U}, n \neq k} w_{n} \ln \operatorname{det}\left(\boldsymbol{R}_{\bar{n}}^{-1} \boldsymbol{R}_{n}\right) .
\end{gathered}
$$

Considering the dependence on the transmitted covariance matrices, only $\mathrm{WR}_{k}^{U L}$ is concave in $\boldsymbol{T}_{k}$, meanwhile $\mathrm{WSR}_{k}^{U} L$ and $\mathrm{WSR}^{D L}$ are non-concave in $\boldsymbol{T}_{k}$, when $\boldsymbol{T}_{\bar{k}}$ and $\boldsymbol{Q}_{j}$, $\forall j \in \mathcal{D}$, are fixed. Similarly, only $\mathrm{WSR}_{j}^{D L}$ is concave in $\boldsymbol{Q}_{j}$ and non-concave in $\operatorname{WSR}_{j}^{D L}$ and $\mathrm{WSR}^{U L}$, when $\boldsymbol{Q}_{\bar{j}}$ and $\boldsymbol{T}_{k}$, $\forall k \in \mathcal{U}$, are fixed. Since a linear function is simultaneously convex and concave, DC programming introduces the first order Taylor series expansion of $\mathrm{WSR}_{\bar{k}}^{U L}$ and $\mathrm{WSR}^{D L}$ in $\boldsymbol{T}_{k}$, around $\hat{\boldsymbol{T}}_{k}$ (i.e. around all $\boldsymbol{T}_{k}$ ), and of $\mathrm{WSR}_{j}^{D} L$ and $\mathrm{WSR}^{U L}$ in $\boldsymbol{Q}_{j}$, around $\hat{\boldsymbol{Q}}_{j}$ (i.e. around all $\boldsymbol{Q}_{j}$ ). Let $\hat{\boldsymbol{T}}$ and $\hat{Q}$ denote the set containing all such $\hat{\boldsymbol{T}}_{k}$ and $\hat{\boldsymbol{Q}}_{j}$, respectively. Let $\hat{\boldsymbol{R}}_{k}(\hat{\boldsymbol{T}}, \hat{\boldsymbol{Q}}), \hat{\boldsymbol{R}}_{\bar{k}}(\hat{\boldsymbol{T}}, \hat{\boldsymbol{Q}}), \hat{\boldsymbol{R}}_{j}(\hat{\boldsymbol{T}}, \hat{\boldsymbol{Q}})$, and $\hat{\boldsymbol{R}}_{\bar{j}}(\hat{\boldsymbol{T}}, \hat{\boldsymbol{Q}})$ denote the covariance matrices $\boldsymbol{R}_{k}, \boldsymbol{R}_{\bar{k}}, \boldsymbol{R}_{j}$ and $\boldsymbol{R}_{\bar{j}}$ as a function of $\hat{\boldsymbol{T}}$ and $\hat{\boldsymbol{Q}}$, respectively. The linearized tangent expression by computing the gradients

$$
\begin{gathered}
\hat{\boldsymbol{A}}_{k}=-\left.\frac{\partial \mathrm{WSR}_{k}^{U}{ }^{L}}{\partial \boldsymbol{T}_{k}}\right|_{\hat{\boldsymbol{T}}, \hat{\boldsymbol{Q}}}, \quad \hat{\boldsymbol{B}}_{k}=-\left.\frac{\partial \mathrm{WSR}^{D L}}{\partial \boldsymbol{T}_{k}}\right|_{\hat{\boldsymbol{T}}, \hat{\boldsymbol{Q}}}, \\
\hat{\boldsymbol{C}}_{j}=-\left.\frac{\partial \mathrm{WSR}_{j}^{D L}}{\partial \boldsymbol{Q}_{j}}\right|_{\hat{\boldsymbol{T}}, \hat{\boldsymbol{Q}}}, \quad \hat{\boldsymbol{D}}_{j}=-\left.\frac{\partial \mathrm{WSR}^{U L}}{\partial \boldsymbol{Q}_{j}}\right|_{\hat{\boldsymbol{T}}, \hat{\boldsymbol{Q}}},
\end{gathered}
$$

with respect to $\boldsymbol{T}_{k}$ and $\boldsymbol{Q}_{j}$ can be written as

$$
\begin{gathered}
\underline{\mathrm{WSR}_{\bar{k}}^{U L}}\left(\boldsymbol{T}_{k}, \hat{\boldsymbol{T}}_{k}\right)=-\operatorname{Tr}\left(\left(\boldsymbol{T}_{\boldsymbol{k}}-\hat{\boldsymbol{T}}_{k}\right) \hat{\boldsymbol{A}}_{k}\right), \\
\underline{\mathrm{WR}^{D L}}\left(\boldsymbol{T}_{k}, \hat{\boldsymbol{T}}_{k}\right)=-\operatorname{Tr}\left(\left(\boldsymbol{T}_{\boldsymbol{k}}-\hat{\boldsymbol{T}}_{k}\right) \hat{\boldsymbol{B}}_{k}\right), \\
\underline{\mathrm{WSR}_{j}^{D L}}\left(\boldsymbol{Q}_{j}, \hat{\boldsymbol{Q}}_{j}\right)=-\operatorname{Tr}\left(\left(\boldsymbol{Q}_{\boldsymbol{j}}-\hat{\boldsymbol{Q}}_{j}\right) \hat{\boldsymbol{C}}_{j}\right), \\
\underline{\mathrm{WSR}^{U L}}\left(\boldsymbol{Q}_{j}, \hat{\boldsymbol{Q}}_{j}\right)=-\operatorname{Tr}\left(\left(\boldsymbol{Q}_{j}-\hat{\boldsymbol{Q}}_{j}\right) \hat{\boldsymbol{D}}_{j}\right) .
\end{gathered}
$$

Note that the linearized tangent expressions $\underline{\mathrm{WSR}_{k}^{U}}{ }^{L}, \underline{\mathrm{WSR}^{D L}}, \mathrm{WSR}_{j}^{D L}$, and $\underline{\mathrm{WSR}^{U L}}$ constitute a touching lower bound for $\mathrm{WSR}_{\frac{k}{k}}^{U L}, \mathrm{WSR} \frac{D}{j}{ }^{L}, \mathrm{WSR}^{D L}$ and $\mathrm{WSR}^{U L}$, respectively. Hence, the DC programming approach is also a minorization-maximization approach, regardless of the restatement of the $\boldsymbol{T}_{k}$ and $\boldsymbol{Q}_{j}$ as a function of the beamformers. The expressions for the gradients in (15a) and (15b) to construct the linearized tangent expressions are given by the following Theorem.

Theorem 1. The gradients $\hat{\boldsymbol{A}}_{k}$ and $\hat{\boldsymbol{B}}_{k}$ which linearize $W S R_{k}^{U L}$ and $W S R^{D L}$, respectively, with respect to $\boldsymbol{T}_{k}, \forall k \in \mathcal{U}$, and the gradients $\hat{\boldsymbol{C}}_{j}$ and $\hat{\boldsymbol{D}}_{j}$ which linearize $W S R_{\bar{j}}^{D L}$ and $W S R^{U L}$, respectively, with respect to $Q_{j}, \forall j \in \mathcal{D}$, with the first order Taylor series expansion, are given by (17).

Proof. Please see Appendix A.

\section{A. Concave Reformulation}

In this section, we proceed by simplifying the non-concave WSR optimization problem (12), by using the results from Theorem 1. By using (17), (12) can be reformulated at each iteration of the alternating optimization process as (18), given at the top of the next page.

Lemma 1. The WSR optimization problem (12) for a single cell multi-antenna multi-user mmWave massive MIMO FD communication system restated at each iteration with its firstorder Taylor series expansion with the gradients (17) as in (18) is a concave reformulation.

Proof. The optimization problem (12) restated as in (18) is made of a concave part i.e. $\log (\cdot)$ and a linear part i.e. $\operatorname{Tr}(\cdot)$. Since a linear function is simultaneously concave and nonconcave, overall (18) results to be concave.

Remark: Note that, the original problem (12) and its reformulation (18) have the same Karush-Kuhn-Tucker (KKT) conditions. Hence, the sub-optimal solution of (18) is also sub-optimal for (12).

Let $\boldsymbol{\Psi}_{0}=\operatorname{diag}\left(\left[\psi_{1}, \ldots, \psi_{M_{0}}\right]\right)$ and $\boldsymbol{\Psi}_{k}=$ $\operatorname{diag}\left(\left[\psi_{k, 1}, \ldots, \psi_{k, M_{k}}\right]\right)$, denote the diagonal matrices containing the Lagrange multiplier associated with the per-antenna power constraints for the FD base station and the uplink users, respectively. Let $l_{0}$ and $l_{1}, \ldots, l_{K}$ denote the Lagrange multipliers associated with the sum-power constraint at the FD base station and at the $K$ uplink users, respectively. We denote with $\boldsymbol{\Psi}$ the collection of all the multipliers associated with the per-antenna power constraints, i.e. $\boldsymbol{\Psi}_{0}$ and $\boldsymbol{\Psi}_{k}, \forall k \in \mathcal{U}$. The collection of all the sum power multipliers are denoted with $\boldsymbol{L}$. Dropping the constant terms, reparameterizing back $\boldsymbol{T}_{k}$ and $\boldsymbol{Q}_{j}$ as a function of beamformers as in (10), performing this linearization for all users in uplink and downlink, and augmenting the WSR cost function with the per-antenna and sum power constraints, yield the Lagrangian (19), given at the top of this page. Note that (19) does not contain the constraints on the analog part, which will be incorporated later.

\section{HYBRID BEAMFORMING AND COMBINING}

This section presents a novel HYBF and combining design for a single cell mmWave massive MIMO FD system based on 


$$
\begin{aligned}
& \hat{\boldsymbol{A}}_{k}=\sum_{i \in \mathcal{U}, i \neq k} w_{i}\left(\boldsymbol{H}_{k}^{H} \boldsymbol{F}_{R F}^{H}\left[\hat{\boldsymbol{R}}_{\bar{i}}(\hat{\boldsymbol{T}}, \hat{\boldsymbol{Q}})^{-1}-\hat{\boldsymbol{R}}_{i}(\hat{\boldsymbol{T}}, \hat{\boldsymbol{Q}})^{-1}-\beta_{0} \operatorname{diag}\left(\hat{\boldsymbol{R}}_{\bar{i}}(\hat{\boldsymbol{T}}, \hat{\boldsymbol{Q}})^{-1}-\hat{\boldsymbol{R}}_{i}(\hat{\boldsymbol{T}}, \hat{\boldsymbol{Q}})^{-1}\right)\right] \boldsymbol{F}_{R F} \boldsymbol{H}_{k}\right. \\
& \left.-k_{i} \operatorname{diag}\left(\boldsymbol{H}_{k}^{H} \boldsymbol{F}_{R F}^{H}\left(\hat{\boldsymbol{R}}_{\bar{i}}(\hat{\boldsymbol{T}}, \hat{\boldsymbol{Q}})^{-1}-\hat{\boldsymbol{R}}_{i}(\hat{\boldsymbol{T}}, \hat{\boldsymbol{Q}})^{-1}\right) \boldsymbol{F}_{R F} \boldsymbol{H}_{k}\right)\right), \\
& \hat{\boldsymbol{B}}_{k}=\sum_{l \in \mathcal{D}} w_{l}\left(\boldsymbol{H}_{l, k}^{H}\left[\hat{\boldsymbol{R}}_{\bar{l}}(\hat{\boldsymbol{T}}, \hat{\boldsymbol{Q}})^{-1}-\hat{\boldsymbol{R}}_{l}(\hat{\boldsymbol{T}}, \hat{\boldsymbol{Q}})^{-1}-\beta_{j} \operatorname{diag}\left(\hat{\boldsymbol{R}}_{\bar{l}}(\hat{\boldsymbol{T}}, \hat{\boldsymbol{Q}})^{-1}-\hat{\boldsymbol{R}}_{l}(\hat{\boldsymbol{T}}, \hat{\boldsymbol{Q}})^{-1}\right)\right] \boldsymbol{H}_{l, k}\right. \\
& \left.-k_{k} \operatorname{diag}\left(\boldsymbol{H}_{l, k}^{H}\left(\hat{\boldsymbol{R}}_{\bar{l}}(\hat{\boldsymbol{T}}, \hat{\boldsymbol{Q}})^{-1}-\boldsymbol{R}_{l}(\hat{\boldsymbol{T}}, \hat{\boldsymbol{Q}})^{-1}\right) \boldsymbol{H}_{l, k}\right)\right)
\end{aligned}
$$

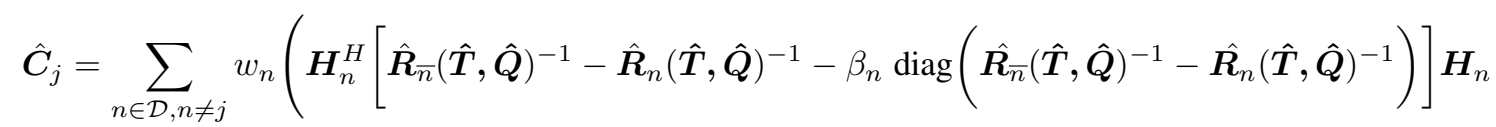

$$
\begin{aligned}
& \left.-k_{0} \operatorname{diag}\left(\boldsymbol{H}_{n}^{H}\left(\hat{\boldsymbol{R}}_{\bar{n}}(\hat{\boldsymbol{T}}, \hat{\boldsymbol{Q}})^{-1}-\hat{\boldsymbol{R}}_{n}(\hat{\boldsymbol{T}}, \hat{\boldsymbol{Q}})^{-1}\right) \boldsymbol{H}_{n}\right)\right), \\
& \left.-k_{0} \operatorname{diag}\left(\boldsymbol{H}_{0}^{H} \boldsymbol{F}_{R F}^{H}\left(\hat{\boldsymbol{R}_{m}}(\hat{\boldsymbol{T}}, \hat{\boldsymbol{Q}})^{-1}-\hat{\boldsymbol{R}}_{m}(\hat{\boldsymbol{T}}, \hat{\boldsymbol{Q}})^{-1}\right) \boldsymbol{F}_{R F} \boldsymbol{H}_{0}\right)\right) \text {, }
\end{aligned}
$$

$$
\begin{array}{ll}
\max _{\substack{\boldsymbol{U}, \boldsymbol{V} \\
\boldsymbol{G}_{R F} \boldsymbol{F}_{R F}}} & \sum_{k \in \mathcal{U}} w_{k} \ln \operatorname{det}\left(\boldsymbol{I}+\boldsymbol{U}_{k}^{H} \boldsymbol{H}_{k}^{H} \boldsymbol{F}_{R F}^{H} \boldsymbol{R}_{\bar{k}}^{-1} \boldsymbol{F}_{R F} \boldsymbol{H}_{k} \boldsymbol{U}_{k}\right)-\operatorname{Tr}\left(\boldsymbol{U}_{k}^{H}\left(\hat{\boldsymbol{A}}_{k}+\hat{\boldsymbol{B}}_{k}\right) \boldsymbol{U}_{k}\right)+ \\
& \sum_{j \in \mathcal{D}} w_{j} \ln \operatorname{det}\left(\boldsymbol{I}+\boldsymbol{V}_{j}^{H} \boldsymbol{G}_{R F}^{H} \boldsymbol{H}_{j}^{H} \boldsymbol{R}_{j}^{-1} \boldsymbol{H}_{j} \boldsymbol{G}_{R F} \boldsymbol{V}_{j}\right)-\operatorname{Tr}\left(\boldsymbol{V}_{j}^{H} \boldsymbol{G}_{R F}^{H}\left(\hat{\boldsymbol{C}}_{j}+\hat{\boldsymbol{D}}_{j}\right) \boldsymbol{G}_{R F} \boldsymbol{V}_{j}\right) \\
\text { s.t. } \quad(12 \mathrm{~b})-(12 \mathrm{~g}) &
\end{array}
$$

$$
\begin{aligned}
\mathcal{L}\left(\boldsymbol{U}, \boldsymbol{V}, \boldsymbol{G}_{R F}, \boldsymbol{F}_{R F}, \boldsymbol{\Psi}, \boldsymbol{L}\right)=\sum_{l=0}^{K} l_{l} \alpha_{l}+\operatorname{Tr}\left(\boldsymbol{\Psi}_{\mathbf{0}} \boldsymbol{\Lambda}_{\mathbf{0}}\right)+\sum_{u \in \mathcal{U}} \operatorname{Tr}\left(\boldsymbol{\Psi}_{\boldsymbol{u}} \boldsymbol{\Lambda}_{\boldsymbol{u}}\right) \\
\quad+\sum_{k \in \mathcal{U}} w_{k} \ln \operatorname{det}\left(\boldsymbol{I}+\boldsymbol{U}_{k}^{H} \boldsymbol{H}_{k}^{H} \boldsymbol{F}_{R F}^{H} \boldsymbol{R}_{\bar{k}}^{-1} \boldsymbol{F}_{R F} \boldsymbol{H}_{k} \boldsymbol{U}_{k}\right)-\operatorname{Tr}\left(\boldsymbol{U}_{k}^{H}\left(\hat{\boldsymbol{A}}_{k}+\hat{\boldsymbol{B}}_{k}+l_{k}+\boldsymbol{\Psi}_{k}\right) \boldsymbol{U}_{k}\right) \\
+\sum_{j \in \mathcal{D}} w_{j} \ln \operatorname{det}\left(\boldsymbol{I}+\boldsymbol{V}_{j}^{H} \boldsymbol{G}_{R F}^{H} \boldsymbol{H}_{j}^{H} \boldsymbol{R}_{j}^{-1} \boldsymbol{H}_{j} \boldsymbol{G}_{R F} \boldsymbol{V}_{j}\right)-\operatorname{Tr}\left(\boldsymbol{V}_{j}^{H} \boldsymbol{G}_{R F}^{H}\left(\hat{\boldsymbol{C}}_{j}+\hat{\boldsymbol{D}}_{j}+l_{0}+\boldsymbol{\Psi}_{0}\right) \boldsymbol{G}_{R F} \boldsymbol{V}_{j}\right)
\end{aligned}
$$

the concave reformulation introduced above. In the following, for the sake of simplified explanation, the design of digital beamformers, analog beamformer and combiner is presented into separate sub-sections. To update one variable, we assume the remaining variables to be fixed during the alternating optimization process. Note that variation in the fixed variables from the previous update is summarized in the gradients, which depend on the covariance matrices. For the analog combiner's update, the design results to be independent from the gradients introduced above. It results to be only a function of the covariance matrices, which are computed based on the most recent update of all the other remaining variables.

\section{A. Digital Beamforming}

To optimize the digital beamformers, assuming the remaining variables to be fixed, we take the derivatives of (19) with respect to the $\boldsymbol{U}_{k}$, which yield the following KKT condition

$$
\begin{gathered}
\boldsymbol{H}_{k}^{H} \boldsymbol{F}_{R F} \boldsymbol{R}_{\bar{k}}^{-1} \boldsymbol{F}_{R F} \boldsymbol{H}_{k} \boldsymbol{U}_{k}\left(\boldsymbol{I}_{u_{k}}+\boldsymbol{U}_{k}^{H} \boldsymbol{H}_{k}^{H} \boldsymbol{F}_{R F}^{H} \boldsymbol{R}_{\bar{k}}^{-1} \boldsymbol{F}_{R F}\right. \\
\left.\boldsymbol{H}_{k} \boldsymbol{U}_{k}\right)^{-1}-\left(\hat{\boldsymbol{A}_{k}}+\hat{\boldsymbol{B}}_{k}+\boldsymbol{\Psi}_{k}+l_{k} \boldsymbol{I}_{u_{k}}\right) \boldsymbol{U}_{k}=0 .
\end{gathered}
$$

Considering the digital beamformers $\boldsymbol{U}_{k}, \forall k$, the analog beamformer and combiner fixed, to optimize $\boldsymbol{V}_{j}$, we take the 
derivative of (19), which yields the following KKT condition

$$
\begin{gathered}
\boldsymbol{G}_{R F}^{H} \boldsymbol{H}_{j}^{H} \boldsymbol{R}_{j}^{-1} \boldsymbol{H}_{j} \boldsymbol{G}_{R F} \boldsymbol{V}_{j}\left(\boldsymbol{I}+\boldsymbol{V}_{j}^{H} \boldsymbol{G}_{R F}^{H} \boldsymbol{H}_{j}^{H} \boldsymbol{R}_{j}^{-1} \boldsymbol{H}_{j} \boldsymbol{G}_{R F}\right. \\
\left.\boldsymbol{V}_{j}\right)^{-1}-\boldsymbol{G}_{R F}^{H}\left(\hat{\boldsymbol{C}}_{j}+\hat{\boldsymbol{D}}_{j}+\boldsymbol{\Psi}_{0}+l_{0} \boldsymbol{I}_{d_{j}}\right) \boldsymbol{G}_{R F} \boldsymbol{V}_{j}=0 .
\end{gathered}
$$

Based on (20a)-(20b), the optimal digital beamformers can be optimized based on the generalized eigenvector solution stated in the following Theorem.

Theorem 2. The optimal digital beamformers $\boldsymbol{U}_{k}, \forall k \in \mathcal{U}$ and $\boldsymbol{V}_{j}, \forall j \in \mathcal{D}$, fixed the analog beamformer and combiner, for (19) are given by the generalized dominant eigenvector solution of the pairs

$$
\begin{array}{r}
\boldsymbol{U}_{k}=\boldsymbol{D}_{u_{k}}\left(\boldsymbol{H}_{k}^{H} \boldsymbol{F}_{R F} \boldsymbol{R}_{\bar{k}}^{-1} \boldsymbol{F}_{R F} \boldsymbol{H}_{k}, \hat{\boldsymbol{A}}_{k}+\hat{\boldsymbol{B}}_{k}+\boldsymbol{\Psi}_{k}+l_{k} \boldsymbol{I}\right) \\
\boldsymbol{V}_{j}=\boldsymbol{D}_{d_{j}}\left(\boldsymbol{G}_{R F}^{H} \boldsymbol{H}_{j}^{H} \boldsymbol{R}_{\bar{j}}^{-1} \boldsymbol{H}_{j} \boldsymbol{G}_{R F}, \boldsymbol{G}_{R F}^{H}\left(\hat{\boldsymbol{C}}_{j}+\hat{\boldsymbol{D}}_{j}+\boldsymbol{\Psi}_{0}\right.\right. \\
\left.\left.+l_{0} \boldsymbol{I}\right) \boldsymbol{G}_{R F}\right),
\end{array}
$$

where $\boldsymbol{D}_{d}(\boldsymbol{X})$ selects the $d$ generalized dominant eigenvectors from the matrix $\boldsymbol{X}$.

Proof. Please see Appendix B.

To include the optimal power control, we normalize the columns of the digital beamformers to be unit-norm. This operation preserves the optimal beamforming directions and enables to design optimal power allocation scheme given the optimal beamforming directions, which is aware of the SI, cross-interference, interference and impairments/LDR based on the values of the gradients $\hat{\boldsymbol{A}}_{k}, \hat{\boldsymbol{B}}_{k}, \hat{\boldsymbol{C}}_{j}, \hat{\boldsymbol{D}}_{j}$.

\section{B. Analog Beamforming}

In contrast to the fully digital beamforming case, the analog beamformer is common to all the downlink users, as shown in Figure 2a-2b. Moreover, its element must have quantized phases and obey the unit-modulus constraint (12f) if there are no AMs available.

Fixed the digital beamformers according to Theorem 2, analog combiner and the multipliers, the unconstrained ana$\log$ beamformer can be optimized by solving the following optimization problem

$$
\begin{gathered}
\max _{\boldsymbol{G}_{R F}} \sum_{j \in \mathcal{D}} w_{j} \ln \operatorname{det}\left(\boldsymbol{I}+\boldsymbol{V}_{j}^{H} \boldsymbol{G}_{R F}^{H} \boldsymbol{H}_{j}^{H} \boldsymbol{R}_{\bar{j}}^{-1} \boldsymbol{H}_{j} \boldsymbol{G}_{R F} \boldsymbol{V}_{j}\right) \\
\quad-\operatorname{Tr}\left(\boldsymbol{V}_{j}^{H} \boldsymbol{G}_{R F}^{H}\left(\hat{\boldsymbol{C}}_{j}+\hat{\boldsymbol{D}}_{j}+l_{0}+\boldsymbol{\Psi}_{0}\right) \boldsymbol{G}_{R F} \boldsymbol{V}_{j}\right)
\end{gathered}
$$

where we have highlighted the structure of the problem, which only depends on the unconstrained analog beamformer. Variation in the digital beamformers and the analog combiner from the previous update is summarized in the gradients. Only the terms shown in (22) depend on the analog beamformer during the alternating optimization process. To optimize the

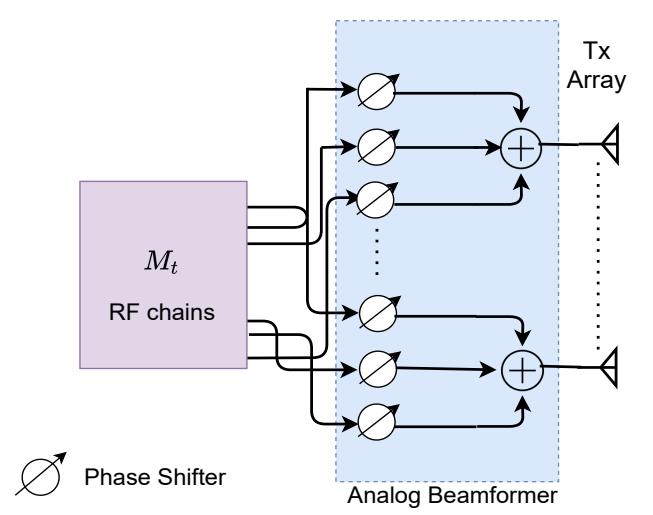

(a) Analog beamformer with unit-modulus phase-shifters.

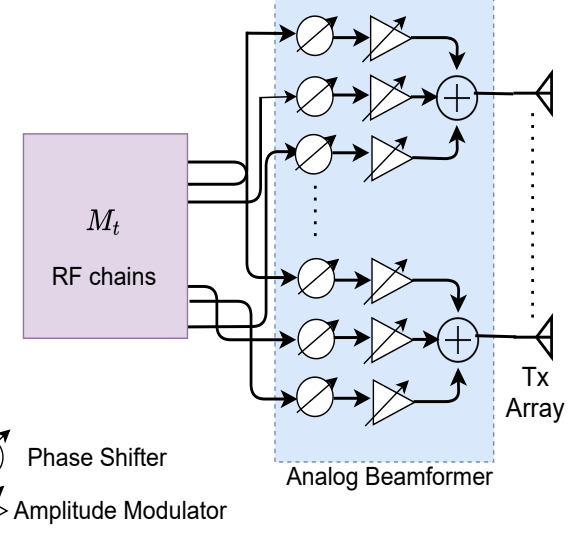

(b) Analog beamformer with amplitude modulators.

Fig. 2: Analog beamformer with a) unit-modulus phase shifter or b) AMs.

analog beamformer, we take the derivative of (22) with respect to $\boldsymbol{G}_{R F}$, which yields the following KKT condition

$$
\begin{gathered}
\boldsymbol{H}_{j}^{H} \boldsymbol{R}_{j}^{-1} \boldsymbol{H}_{j} \boldsymbol{G}_{R F} \boldsymbol{V}_{j} \boldsymbol{V}_{j}^{H}\left(\boldsymbol{I}+\boldsymbol{V}_{j} \boldsymbol{V}_{j}^{H} \boldsymbol{G}_{R F}^{H} \boldsymbol{H}_{j}^{H} \boldsymbol{R}_{j}^{-1} \boldsymbol{H}_{j}\right. \\
\left.\boldsymbol{G}_{R F}\right)^{-1}-\left(\hat{\boldsymbol{C}}_{j}+\hat{\boldsymbol{D}}_{j}+\boldsymbol{\Psi}_{0}+l_{0} \boldsymbol{I}\right) \boldsymbol{G}_{R F} \boldsymbol{V}_{j} \boldsymbol{V}_{j}^{H}=0 .
\end{gathered}
$$

The optimal analog beamformer, common to all the downlink users, can be optimized based on the result stated in the following.

Theorem 3. The optimal vectorized unconstrained analog beamformer vec $\left(\boldsymbol{G}_{R F}\right)$ which solves (22) is given by the generalized dominant eigenvector solution of the pairs

$$
\begin{aligned}
\operatorname{vec}\left(\boldsymbol{G}_{R F}\right) & =\boldsymbol{D}_{1}\left(\sum _ { j \in \mathcal { D } } \left(\boldsymbol { V } _ { j } \boldsymbol { V } _ { j } ^ { H } \left(\boldsymbol{I}+\boldsymbol{V}_{j} \boldsymbol{V}_{j}^{H} \boldsymbol{G}_{R F}^{H} \boldsymbol{H}_{j}^{H} \boldsymbol{R}_{j}^{-1}\right.\right.\right. \\
& \left.\left.\boldsymbol{H}_{j} \boldsymbol{G}_{R F}\right)^{-1}\right)^{T} \otimes \boldsymbol{H}_{j}^{H} \boldsymbol{R}_{\bar{j}}^{-1} \boldsymbol{H}_{j}, \\
& \left.\sum_{j \in \mathcal{D}}\left(\boldsymbol{V}_{j} \boldsymbol{V}_{j}^{H}\right)^{T} \otimes\left(\hat{\boldsymbol{C}}_{j}+\hat{\boldsymbol{D}}_{j}+\boldsymbol{\Psi}_{0}+l_{0} \boldsymbol{I}\right)\right),
\end{aligned}
$$

where $\boldsymbol{D}_{1}(\boldsymbol{X})$ selects the first generalized dominant eigenvectors of the matrix $\boldsymbol{X}$. 
Proof. Please see Appendix B.

Note that Theorem 3 provides the optimal solution for vectorized unconstrained analog beamformer $\boldsymbol{G}_{R F}$, therefore we need the operation unvec $\left(\boldsymbol{G}_{R F}\right)$ to reshape the analog beamformer into correct dimensions. To render a solution that satisfy the unit-modulus constraints, we perform the operation $\boldsymbol{G}_{R F}=\angle \boldsymbol{G}_{R F}$ and pass its elements to the quantizer $\mathbb{Q}(\cdot)$ such that $\boldsymbol{G}_{R F}(m, n) \in \mathcal{P}, \forall m, n$ with $a_{i}=1$ for $0 \leq i \leq n_{p s}-1$. If the AMs are available, both the phase and the amplitude part are passed through $\mathbb{Q}(\cdot)$ such that $\boldsymbol{G}_{R F}(m, n) \in \mathcal{P}, \forall m, n$ with any value of $a_{i}$ for $0 \leq i \leq n_{p s}-1$

\section{Analog Combiner}

In this section, we consider the optimization of analog combiner $\boldsymbol{F}_{R F}$, fixed the analog and digital beamformers and the multipliers. Note that in the simplified problem based on minorization-maximization, the analog combiner does not appear in the trace operator. The trace term, with the gradients make the beamformers update aware of the interference generated towards the other links and thus limits greedy behaviour. Fixed other variables, for the analog combiner optimization, we can directly consider the original optimization problem (12), which is concave for the analog combiner and depends only on the covariance matrices (function of the digital beamformers, analog beamformer and impairments).

Let us consider first the unconstrained analog combiner, i.e.

$$
\max _{\boldsymbol{F}_{R F}} \sum_{k \in \mathcal{U}} w_{k} \ln \operatorname{det}\left(\boldsymbol{R}_{\bar{k}}^{-1} \boldsymbol{R}_{k}\right) .
$$

Let $\left(\boldsymbol{R}_{k}^{a n t}\right) \boldsymbol{R}_{\bar{k}}^{a n t}$ denote the (signal-plus) interference and noise covariance matrix received at the antenna of the FD base station before the analog combining stage, which can be obtained from $\boldsymbol{R}_{k}$ and $\boldsymbol{R}_{\bar{k}}$ (11), without $\boldsymbol{F}_{R F}$. After the analog combining stage, we have $\boldsymbol{R}_{k}=\boldsymbol{F}_{R F} \boldsymbol{R}_{k}^{a n t} \boldsymbol{F}_{R F}^{H}$ and $\boldsymbol{R}_{\bar{k}}=\boldsymbol{F}_{R F} \boldsymbol{R}_{\bar{k}}^{a n t} \boldsymbol{F}_{R F}^{H}, \forall k \in \mathcal{U}$ (see (11)). The WSR maximization problem (25) can be written as

$$
\begin{aligned}
\max _{\boldsymbol{F}_{R F}} \sum_{k \in \mathcal{U}}[ & w_{k} \ln \operatorname{det}\left(\boldsymbol{F}_{R F} \boldsymbol{R}_{k}^{a n t} \boldsymbol{F}_{R F}^{H}\right) \\
& \left.-w_{k} \ln \operatorname{det}\left(\boldsymbol{F}_{R F} \boldsymbol{R}_{\bar{k}}^{a n t} \boldsymbol{F}_{R F}^{H}\right)\right] .
\end{aligned}
$$

Note that (26) has the same structure of the minorizationmaximization problem, where the trace term was linear, thus making the restated optimization problem concave, fixed the other variables. Similarly, for the analog combiner update, we have a (fully) concave optimization problem. To optimize the analog combiner, we take the derivative with respect to $\boldsymbol{F}_{R F}$ of (26), which yield the following KKT conditions

$$
\begin{aligned}
\sum_{k \in \mathcal{U}} w_{k} & \boldsymbol{R}_{k}^{a n t} \boldsymbol{F}_{R F}\left(\boldsymbol{F}_{R F} \boldsymbol{R}_{k}^{a n t} \boldsymbol{F}_{R F}^{H}\right)^{-1} \\
& -\sum_{k \in \mathcal{U}} w_{k} \boldsymbol{R}_{\bar{k}}^{a n t} \boldsymbol{F}_{R F}\left(\boldsymbol{F}_{R F} \boldsymbol{R}_{\bar{k}}^{a n t} \boldsymbol{F}_{R F}^{H}\right)^{-1}=0 .
\end{aligned}
$$

It is immediate to see that the optimal unconstrained analog combiner is given by the dominant generalized eigenvectors of the pair of the $\boldsymbol{R}_{k}^{a n t}$ and $\boldsymbol{R}_{\bar{k}}^{a n t}$, summed over all the uplink users, i.e.,

$$
\boldsymbol{F}_{R F} \rightarrow \boldsymbol{D}_{N 0}\left(w_{k} \sum_{k \in \mathcal{U}} \boldsymbol{R}_{k}^{a n t}, w_{k} \sum_{k \in \mathcal{U}} \boldsymbol{R}_{\bar{k}}^{a n t}\right)
$$

from which we select $N_{r}$ rows. To render a solution which satisfy the unit-modulus constraints, we perform the operation $\boldsymbol{F}_{R F}=\angle \boldsymbol{F}_{R F}$ and pass its elements by the quantizer $\mathbb{Q}(\cdot)$ with $a_{i}=1, \forall i$. If the AMs are available, both the phase and the amplitude part are quantized, such that $\boldsymbol{F}_{R F}(m, n) \in \mathcal{P}$, $\forall m, n$ with $a_{i} \neq 1$.

\section{Optimal Power Allocation}

After computing the optimal normalized digital beamformers and the analog beamformer, the optimal power allocation can be included while searching for the Lagrange multipliers satisfying the joint sum-power and per-antenna power constraints.

Fixed the beamformers, let

$$
\begin{gathered}
\boldsymbol{U}_{k}^{H} \boldsymbol{H}_{k}^{H} \boldsymbol{F}_{R F}^{H} \boldsymbol{R}_{k}^{-1} \boldsymbol{F}_{R F} \boldsymbol{H}_{k} \boldsymbol{U}_{k}=\boldsymbol{\Sigma}_{k}^{(1)} \\
\boldsymbol{U}_{k}^{H}\left(\hat{\boldsymbol{A}}_{k}+\hat{\boldsymbol{B}}_{k}+\boldsymbol{\Psi}_{k}+l_{k} \boldsymbol{I}\right) \boldsymbol{U}_{k}=\boldsymbol{\Sigma}_{k}^{(2)} \\
\boldsymbol{V}_{j}^{H} \boldsymbol{G}_{R F}^{H} \boldsymbol{H}_{j}^{H} \boldsymbol{R}_{j}^{-1} \boldsymbol{H}_{j} \boldsymbol{G}_{R F} \boldsymbol{V}_{j}=\boldsymbol{\Sigma}_{j}^{(1)} \\
\boldsymbol{V}_{j}^{H} \boldsymbol{G}_{R F}^{H}\left(\hat{\boldsymbol{C}}_{j}+\hat{\boldsymbol{D}}_{j}+\boldsymbol{\Psi}_{0}+l_{0} \boldsymbol{I}\right) \boldsymbol{G}_{R F} \boldsymbol{V}_{j}=\boldsymbol{\Sigma}_{j}^{(2)} .
\end{gathered}
$$

Given the optimal beamformers and fixed Lagrange multipliers, the optimal power allocation can be included relying on the result stated in Lemma 2 in the following.

Lemma 2. The optimal power allocation for the FD base station and HD multi-antenna uplink users can be obtained by multiplying $\boldsymbol{\Sigma}_{k}^{(1)}$ and $\boldsymbol{\Sigma}_{k}^{(2)}$ with diagonal power matrix $\boldsymbol{P}_{k}$, $\forall k \in \mathcal{U}$ and $\boldsymbol{\Sigma}_{j}^{(1)}$ and $\boldsymbol{\Sigma}_{j}^{(2)}$ with diagonal power matrix $\boldsymbol{P}_{j}$, $\forall j \in \mathcal{D}$.

Proof. The optimal beamformers $U_{k}, V_{k}, G_{R F}$ are the generalized dominant eigenvector solution, thus making the matrices $\boldsymbol{\Sigma}_{k}^{(1)}, \boldsymbol{\Sigma}_{k}^{(2)}, \boldsymbol{\Sigma}_{j}^{(1)}$ and $\boldsymbol{\Sigma}_{j}^{(2)}$ diagonal. Multiplying any generalized dominant eigenvector solution with a diagonal matrix, still yields a generalized dominant eigenvector solution. Therefore, multiplying $\boldsymbol{\Sigma}_{k}^{(1)}, \boldsymbol{\Sigma}_{k}^{(2)}$ with $\boldsymbol{P}_{k}, \forall k \in \mathcal{U}$ and $\boldsymbol{\Sigma}_{j}^{(1)}, \boldsymbol{\Sigma}_{j}^{(2)}$ with $\boldsymbol{P}_{j}, \forall j \in \mathcal{D}$ still preserves the validity of the generalized dominant eigenvector solutions.

Given the optimal beamformers and fixed Lagrange multipliers, the power allocation optimization problem for the uplink and the downlink users can be stated formally as

$$
\begin{aligned}
& \max _{\boldsymbol{P}_{k}} w_{k} \ln \operatorname{det}\left(\boldsymbol{I}+\boldsymbol{\Sigma}_{k}^{(1)} \boldsymbol{P}_{k}\right)-\operatorname{Tr}\left(\boldsymbol{\Sigma}_{k}^{(2)} \boldsymbol{P}_{k}\right), \quad \forall k \in \mathcal{U}, \\
& \max _{\boldsymbol{P}_{j}} w_{j} \ln \operatorname{det}\left(\boldsymbol{I}+\boldsymbol{\Sigma}_{j}^{(1)} \boldsymbol{P}_{j}\right)-\operatorname{Tr}\left(\boldsymbol{\Sigma}_{j}^{(2)} \boldsymbol{P}_{j}\right), \quad \forall j \in \mathcal{D} .
\end{aligned}
$$


Optimizing powers for each transmission link in both the uplink and downlink directions yield

$$
\begin{gathered}
\boldsymbol{P}_{k}=\left(w_{k}\left(\boldsymbol{U}_{k}^{H}\left(\hat{\boldsymbol{A}}_{k}+\hat{\boldsymbol{B}}_{k}+\boldsymbol{\Psi}_{k}+l_{k} \boldsymbol{I}\right) \boldsymbol{U}_{k}\right)^{-1}\right. \\
\left.-\left(\boldsymbol{U}_{k}^{H} \boldsymbol{H}_{k}^{H} \boldsymbol{F}_{R F}^{H} \boldsymbol{R}_{\bar{k}}^{-1} \boldsymbol{F}_{R F} \boldsymbol{H}_{k} \boldsymbol{U}_{k}\right)^{-1}\right)^{+} \\
\boldsymbol{P}_{j}=\left(w_{j}\left(\boldsymbol{V}_{j}^{H} \boldsymbol{G}_{R F}^{H}\left(\hat{\boldsymbol{C}}_{j}+\hat{\boldsymbol{D}}_{j}+\boldsymbol{\Psi}_{0}+l_{0} \boldsymbol{I}\right) \boldsymbol{G}_{R F} \boldsymbol{V}_{j}\right)^{-1}\right. \\
\left.-\left(\boldsymbol{V}_{j}^{H} \boldsymbol{G}_{R F}^{H} \boldsymbol{H}_{j}^{H} \boldsymbol{R}_{j}^{-1} \boldsymbol{H}_{j} \boldsymbol{G}_{R F} \boldsymbol{V}_{j}\right)^{-1}\right)^{+}
\end{gathered}
$$

where $(\boldsymbol{X})^{+}=\max \{0, \boldsymbol{X}\}$. We remark the the proposed power allocation scheme is interference, SI, cross-interference and impairments/LDR aware as it takes into account their effect in the gradients at each iteration of the alternating optimization process. Fixed the optimal beamformers given by the generalized dominant eigenvector solution, we can search for the optimal Lagrange multipliers satisfying the per-antenna and sum-power constraints while performing water-filling for the powers. Consider the dependence of the Lagrangian associated with the concave reformulation on the multipliers and powers

$$
\begin{aligned}
& \mathcal{L}(\boldsymbol{\Psi}, \boldsymbol{L}, \boldsymbol{P})=\sum_{l=0}^{K} l_{l} p_{l}+\operatorname{Tr}\left(\boldsymbol{\Psi}_{\mathbf{0}} \boldsymbol{\Lambda}_{\mathbf{0}}\right)+\sum_{u \in \mathcal{U}} \operatorname{Tr}\left(\boldsymbol{\Psi}_{\boldsymbol{u}} \boldsymbol{\Lambda}_{\boldsymbol{u}}\right) \\
& +\sum_{k \in \mathcal{U}} w_{k} \ln \operatorname{det}\left(\boldsymbol{I}+\boldsymbol{\Sigma}_{k}^{(1)} \boldsymbol{P}_{k}\right)-\operatorname{Tr}\left(\boldsymbol{\Sigma}_{k}^{(2)} \boldsymbol{P}_{k}\right) \\
& +\sum_{j \in \mathcal{D}} w_{j} \ln \operatorname{det}\left(\boldsymbol{I}+\boldsymbol{\Sigma}_{j}^{(1)} \boldsymbol{P}_{j}\right)-\operatorname{Tr}\left(\boldsymbol{\Sigma}_{j}^{(2)} \boldsymbol{P}_{j}\right),
\end{aligned}
$$

where $\boldsymbol{P}$ is the set of all stream powers in uplink and downlink. The multipliers $\boldsymbol{\Psi}$ and $\boldsymbol{L}$ should be such that the Lagrange dual function (32) is finite and the values of the multipliers should be strictly positive. Formally, the multipliers' search problem can be stated as

$$
\begin{array}{rr}
\min _{\boldsymbol{\Psi}, \boldsymbol{L}} \max _{\boldsymbol{P}} & \mathcal{L}(\boldsymbol{\Psi}, \boldsymbol{L}, \boldsymbol{P}), \\
\text { s.t. } & \boldsymbol{\Psi}, \boldsymbol{L} \succeq 0 .
\end{array}
$$

The dual function $\max _{\boldsymbol{P}} \mathcal{L}(\boldsymbol{\Psi}, \boldsymbol{L}, \boldsymbol{P})$ is the pointwise supremum of a family of functions of $\boldsymbol{\Psi}, \boldsymbol{L}$, it is convex [56] and the globally optimal values for $\boldsymbol{\Psi}$ and $\boldsymbol{L}$ can be found by using any of the numerous convex optimization techniques. In this work, we adopt the bisection algorithm to search for the multipliers. Let $\mathcal{M}_{0}=\left\{\lambda_{0}, \psi_{1}, . ., \psi_{M 0}\right\}$ and $\mathcal{M}_{k}=\left\{\lambda_{k}, \psi_{k, 1}, . ., \psi_{k, M_{k}}\right\}$ denote the set containing the Lagrange multipliers associated with the sum-power and per-antenna power constraint at the FD base station and the uplink user $k \in \mathcal{U}$. Let $\underline{\mu_{i}}$ and $\overline{\mu_{i}}$ be the upper and lower bounds for the search range defined for the multiplier $\mu_{i}, \forall i \in \mathcal{M}_{k}, \forall k \in \mathcal{U}$ or $\forall i \in \mathcal{M}_{0}$. Note that after the search of multipliers while doing water-filling, the uplink and downlink power matrices are not diagonal anymore. Therefore, we consider the singular value decomposition (SVD) of the power matrices to obtain the diagonal power matrices again. Namely, let $\boldsymbol{P}_{i}$ denote the power matrix, where

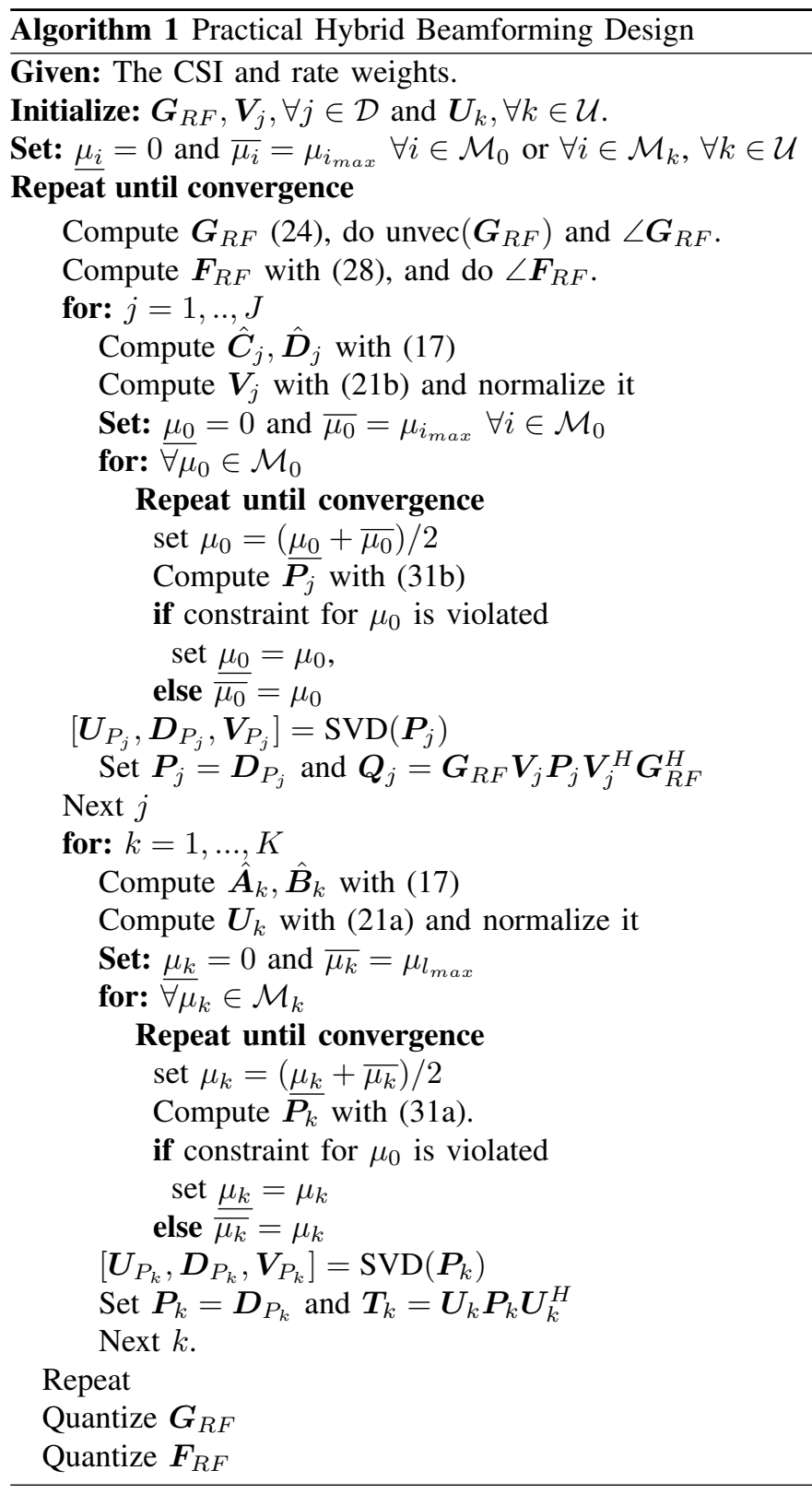

$i \in \mathcal{U}$ or $\mathcal{D}$. The powers can be updated while searching for the multipliers as

$$
\begin{gathered}
{\left[\boldsymbol{U}_{P_{i}}, \boldsymbol{D}_{P_{i}}, \boldsymbol{V}_{P_{i}}\right]=\operatorname{SVD}\left(\boldsymbol{P}_{i}\right),} \\
\boldsymbol{P}_{i}=\boldsymbol{D}_{P_{i}},
\end{gathered}
$$

where $\boldsymbol{U}_{P_{i}}, \boldsymbol{D}_{P_{i}}, \boldsymbol{V}_{P_{i}}$ are the left unitary, diagonal and right unitary matrices obtained with the SVD decomposition.

The alternating optimization based algorithm for hybrid beamforming and combining in a single-cell mmWave massive MIMO FD system based on the generalized dominant eigenvector solution is given in Algorithm 1.

\section{E. Convergence}

In our context, the ingredients required to prove the convergence are minorization [46], alternating or cyclic optimization [46] (also called block coordinate descent), Lagrange dual 
function [57], saddle-point interpretation [57] and KKT conditions [57]. For the WSR cost function (12), we construct its minorizer as in (16a), (16b), (16c), (16d), which restates the WSR maximization as a concave problem (18). The minorizer results to be a touching lower bound for the original WSR problem, therefore we can write

$$
\mathrm{WSR} \geq \underline{\mathrm{WSR}}=\underline{\mathrm{WR}_{k}^{U L}}+\underline{\mathrm{WSR}_{\bar{k}}^{U L}}+\underline{\mathrm{WR}_{j}^{D L}}+\underline{\mathrm{WSR}_{j}^{D L}} .
$$

The minorizer, which is concave in $\boldsymbol{T}_{k}$ and $\boldsymbol{Q}_{j}$, still has the same gradient of the original WSR and hence the KKT conditions are not affected. Now reparameterizing $\boldsymbol{T}_{k}$ or $\boldsymbol{Q}_{j}$ in terms of $\boldsymbol{F}_{R F}, \boldsymbol{G}_{R F}, \boldsymbol{V}_{j}, \forall j \in \mathcal{D}$ or $\boldsymbol{U}_{k}, \forall k \in \mathcal{U}$, respectively as in (10) with the optimal power matrices and adding the power constraints to the minorizer, we get the Lagrangian (19). Every alternating update of $\mathcal{L}$ for $\boldsymbol{V}_{j}, \boldsymbol{G}_{R F}$, $\boldsymbol{U}_{k}, \forall j \in \mathcal{D}, \forall k \in \mathcal{U}$ or for $\boldsymbol{P}, \boldsymbol{\Lambda}, \boldsymbol{\Psi}$ leads to an increase of the WSR, ensuring convergence. For the KKT conditions, at the convergence point, the gradients of $\mathcal{L}$ for $\boldsymbol{V}_{j}$ or $\boldsymbol{G}_{R F}$ or $\boldsymbol{U}_{j}$ or $\mathbf{P}$ correspond to the gradients of the Lagrangian of the original WSR (12). For fixed analog and the digital beamformers, $\mathcal{L}$ is concave in $\mathbf{P}$, hence we have strong duality for the saddle point, i.e.

$$
\max _{\mathbf{P}} \min _{\boldsymbol{L}, \boldsymbol{\Psi}} \mathcal{L}(\boldsymbol{L}, \boldsymbol{\Psi}, \mathbf{P}) .
$$

Let $\boldsymbol{X}^{*}$ and $x^{*}$ denote the optimal solution for matrix $\boldsymbol{X}$ or scalar $x$ at the convergence, respectively. At the convergence point, the solution of the optimization problem

$$
\min _{\boldsymbol{\Lambda}, \Psi} \mathcal{L}\left(\mathbf{V}^{*}, \mathbf{G}^{*}, \mathbf{U}^{*}, \mathbf{P}^{*}, \mathbf{L}, \Psi\right)
$$

satisfies the KKT conditions for the powers in $\mathbf{P}$ and the complementary slackness conditions

$$
\begin{gathered}
l_{0}^{*}\left(\alpha_{0}-\sum_{j \in \mathcal{D}} \operatorname{Tr}\left(\boldsymbol{G}_{R F}^{*} \boldsymbol{V}_{j}^{*} \mathbf{P}_{j}^{*} \mathbf{V}_{j}^{* H} \boldsymbol{G}_{R F}^{* H}\right)\right)=0 \\
\operatorname{Tr}\left(\boldsymbol{\Psi}_{0}^{*}\left(\mathbf{P}_{0}-\sum_{j \in \mathcal{D}} \operatorname{Tr}\left(\boldsymbol{G}_{R F}^{*} \mathbf{V}_{j}^{*} \mathbf{P}_{j}^{*} \mathbf{V}_{j}^{* H} \boldsymbol{G}_{R F}^{* H}\right)\right)\right)=0 \\
l_{k}^{*}\left(\alpha_{k}-\operatorname{Tr}\left(\mathbf{U}_{k}^{*} \mathbf{P}_{k}^{*} \mathbf{U}_{k}^{* H}\right)\right)=0 \\
\operatorname{Tr}\left(\mathbf{\Psi}_{k}^{*}\left(\mathbf{P}_{k}-\operatorname{Tr}\left(\mathbf{U}_{k}^{*} \mathbf{P}_{k}^{*} \mathbf{U}_{k}^{* H}\right)\right)\right)=0
\end{gathered}
$$

where all the individual factors in the products are nonnegative and for the per-antenna power constraints $\Psi_{0}^{*}$ and $\boldsymbol{\Psi}_{k}^{*}$, the sum of non-negative terms being zero implies all the terms result to be zero.

\section{F. Complexity Analysis}

In this section, we analyze the per-iteration computational complexity for the proposed HYBF design, in terms of the number of complex multiplications. In Algorithm 1, one dominant generalized eigenvector computation of the analog beamformer from matrix of size $M_{t} M_{0} \times M_{t} M_{0}$ in (24), is $\mathcal{O}\left(M_{0}^{2} M_{t}^{2}\right)$. The uplink and downlink digital beamformer update has additional computational complexity of $\mathcal{O}\left(u_{k} M_{k}^{2}\right)$

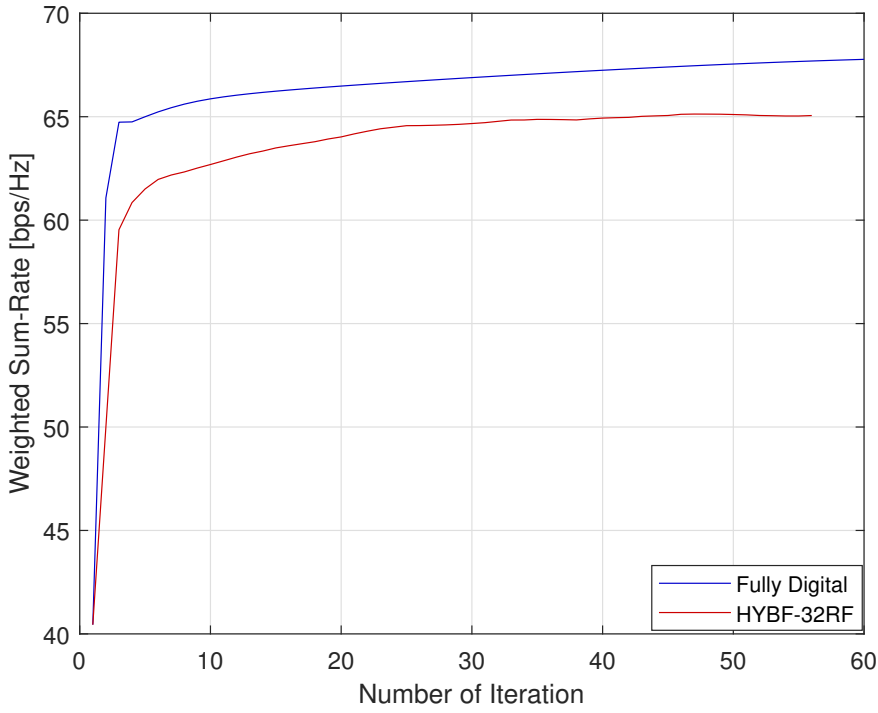

Fig. 3: Typical convergence behaviour of the proposed HYBF and combining design.

and $\mathcal{O}\left(v_{j} N_{j}^{2}\right)$, respectively. To update the gradient $\hat{\mathbf{A}}_{k}$, it involves a matrix inversion $\left(\mathbf{R}_{\bar{k}}^{-1}\right)$ which has complexity $\mathcal{O}\left(N_{r}^{3}\right)$. Considering the total number of multiplications, and inversion, $\hat{\mathbf{A}}_{k}$ has the total complexity $\mathcal{O}\left(N_{r}^{3}\right)+\mathcal{O}\left(M_{k} N_{r}^{2}\right)+$ $\mathcal{O}\left(M_{k}^{2} N_{r}\right)$. Similarly, to compute $\hat{\mathbf{B}}_{k}$, the total complexity is $\mathcal{O}\left(N_{k}^{3}\right)+\mathcal{O}\left(M_{k} N_{k}^{2}\right)+\mathcal{O}\left(M_{k}^{2} N_{k}\right)$. For the gradient $\widehat{\mathbf{C}}_{j}$ which is part of the downlink beamformer's update, the matrix inversion $\left(\mathbf{R}_{\bar{j}}^{-1}\right)$ is required which is $\mathcal{O}\left(N_{j}^{3}\right)$. By considering also the matrix multiplications, the total complexity involved for $\hat{\mathbf{C}}_{j}$ is $\mathcal{O}\left(N_{j}^{3}\right)+\mathcal{O}\left(M_{0} N_{j}^{2}\right)+\mathcal{O}\left(M_{0}^{2} N_{j}\right)$. Similarly, the total complexity involved in $\hat{\mathbf{D}}_{j}$ results to be $\mathcal{O}\left(N_{r}^{3}\right)+\mathcal{O}\left(M_{0} N_{r}^{2}\right)+$ $\mathcal{O}\left(M_{0}^{2} N_{r}\right)$. The Lagrange multipliers updated associated with the per-antenna power constraints at the FD base station or the uplink users, is linear in the number of antennas $M_{0}$ or $M_{k}$, respectively. However, as we jointly perform the multipliers' search and the power matrix computations in (34a), it adds an additional complexity of $\mathcal{O}\left(v_{i}^{3}\right)$, where $i \in \mathcal{D}$ or $i \in \mathcal{U}$, due to the SVD. For the analog combiner at the base station, the generalized eigenvector computations is $\mathcal{O}\left(N_{0}^{3}\right)$. Assuming that the dimensions of the antennas get large, the overall complexity of the proposed algorithm is given by $\approx \mathcal{O}\left(2 N_{r}^{3}+N_{k}^{3}+N_{j}^{3}+N_{0}^{3}+M_{0}^{2} M_{t}^{2}\right)$.

\section{Simulation Results}

This section presents simulation results to evaluate the performance for the proposed practical HYBF and combining scheme. For comparison, we define the following benchmark schemes:

a) A Fully digital HD-ideal hardware approach, having the number of radio frequency chains equal to the number of antennas and serving the users by switching the uplink and downlink mode by separating the resources in time. As the uplink and downlink users are not served simultaneously, we have neither SI nor the cross-interference generated from the uplink users towards the downlink users. We are interested in 
evaluating how much additional gain we can still achieve with the proposed HYBF design with different levels of the LDR noise compared to the maximum achievable performance of a HD system. Therefore, we consider a HD system with no LDR noise.

b) A Fully digital FD-ideal hardware scheme, having the number of radio frequency chains equal to the number of antennas with no LDR noise. This scheme set an upper bound on the maximum achievable performance for an FD system in mmWave.

c) A Fully digital FD scheme, having the same characteristics as scheme b), but affected with the LDR noise.

The proposed joint HYBF and combining design is denoted with HYBF-UM or HYBF-AM for the analog processing stage with unit-modulus constraint or with AMs, respectively. We define the signal-to-noise-ratio (SNR) of the mmWave hybrid FD system as

$$
\mathrm{SNR}=\alpha_{0} / \sigma_{0}^{2}=\alpha_{k} / \sigma_{k}^{2},
$$

where $\alpha_{0}$ and $\alpha_{k}$ is the total transmit power for the base station and uplink users, respectively, $\sigma_{0}^{2}$ and $\sigma_{k}^{2}$ denote the noise variance at the FD node and at the uplink user $k \in \mathcal{U}$, respectively. We consider the total transmit power to be normalized to 1 and choose the noise variances values based on the desired SNR. To compare the gain of a FD system over a HD system, we define the additional gain in percentage as

$$
\text { Gain }=\frac{W S R_{F D}-W S R_{H D}}{W S R_{H D}} \times 100[\%],
$$

where $W S R_{F D}$ and $W S R_{H D}$ denote the WSR of an FD and a HD system, respectively. To evaluate the performance, we set the per antenna power constraints for the FD and uplink users as the total transmit power divided by the total number of antennas, i.e. $\alpha_{0} / M_{0} \mathbf{I}$ and $\alpha_{k} / M_{k} \mathbf{I}, \forall k$. The base station and users are assumed to be equipped with a uniform linear array (ULA) with antennas separated by half-wavelength. The transmit and receive antenna array at the base station are assumed to be placed $D=20 \mathrm{~cm}$ apart with the angle between the transmit and receive array $\Theta=90^{\circ}$, and the distance parameter is modelled as (9) [19]. The Rician factor $\kappa$ for the SI channel is set to be 1 . We assume that the FD base station has $M_{0}=100$ transmit and $N_{0}=50$ receive antennas and users have $M_{k}=N_{j}=5$ antennas, and served with 2 data streams each. We assume that the phase-shifters at the analog processing stage to be quantized with a 8 bit uniform quantizer. Note that as we are assuming perfect CSI, the SI can be cancelled with HYBF up to the LDR noise level only (which is the residual SI). The rate-weights for all the users are set to be 1 . All the simulation parameters are summarized in Table II. The digital beamformers are intialized as dominant eigen vectors of the channel covariance matrices of the intended users. The analog beamformer and combiner are initialized as dominant eigen vectors of the sum of channel covariance
TABLE II: Simulation parameters choice to simulate the practical mmWave massive MIMO FD system.

\begin{tabular}{|l|l|l|}
\hline \multicolumn{3}{|c|}{ Simulation Parameters } \\
\hline UL and DL users & $K, J$ & 2 \\
\hline Data streams & $d_{j}, u_{k}$ & 2 \\
\hline Tx antenna (BS) & $M_{0}$ & 100 \\
\hline Rx antenna (BS) & $N_{0}$ & 50 \\
\hline Clusters and Paths & $N_{c}, N_{p}$ & 3 \\
\hline $\begin{array}{l}\text { Tx and Rx RF } \\
\text { chains }\end{array}$ & $M_{t}=N_{r}$ & $8,10,16$ or 32 \\
\hline User antennas & $M_{k}=N_{j}$ & 5 \\
\hline Rician Factor & $\kappa$ & 1 \\
\hline $\begin{array}{l}\text { Tx and Rx array } \\
\text { response (BS) }\end{array}$ & $\boldsymbol{a}_{r}, \boldsymbol{a}_{t}$ & $\mathrm{ULA}$ \\
\hline Angles & $\phi_{k}, \phi_{j}, \theta_{k}, \theta_{j}$ & $\mathcal{U} \sim\left[-30^{\circ}, 30^{\circ}\right]$ \\
\hline Rate weights & $w_{k}, w_{j}$ & 1 \\
\hline $\begin{array}{l}\text { Uniform } \\
\text { Quantizer }\end{array}$ & $\left.\mathbb{Q}^{\circ} \cdot\right)$ & 8 bit \\
\hline $\begin{array}{l}\text { Angle between Tx } \\
\text { and Rx array at } \\
\text { the BS }\end{array}$ & $\Theta$ & $90^{\circ}$ \\
\hline $\begin{array}{l}\text { Distance between } \\
\text { Tx and Rx array } \\
\text { at the BS }\end{array}$ & $D$ & $\alpha_{0} / M_{0} \boldsymbol{I}$ \\
\hline $\begin{array}{l}\text { Per-antenna } \\
\text { power constraint } \\
\text { for UL user } k\end{array}$ & $\boldsymbol{\Lambda}_{k}$ & $20 \mathrm{~cm}$ \\
\hline $\begin{array}{l}\text { Per-antenna } \\
\text { power constraint } \\
\text { for the BS }\end{array}$ & $\boldsymbol{\Lambda}_{0}$ & $\alpha_{k} / M_{k} \boldsymbol{I}$ \\
\hline
\end{tabular}

matrices across all the downlink and uplink users, respectively.

Figure 4 shows the performance of the proposed joint HYBF and combining design, in terms of average WSR, as a function of $k_{0}=\beta_{0}=\kappa_{k}=\beta_{j}$, when the SNR is $20 \mathrm{~dB}$ with 32 or $16 \mathrm{RF}$ chains. We can see that the fully digital FD scheme achieves an additional gain of $95 \%$ over a fully digital HD scheme. We can also see the impact of different LDR noise levels on the achievable performance of an FD system in mmWave. It can be that at $\mathrm{SNR}=20 \mathrm{~dB}$, the proposed $\mathrm{HYBF}$ design with unit-modulus phase-shifters achieves around $\sim 67 \%$ additional gain with only $16-\mathrm{RF}$ chains and around $\sim 81.43 \%$ with $32-\mathrm{RF}$ chains for $k_{0}=$ $\beta_{0}=\kappa_{k}=\beta_{j} \leq-60 \mathrm{~dB}$. Amplitude modulators add an additional gain of $\sim 1.2 \%$ and $\sim 7.6 \%$ compared to the unit-modulus scheme with 32 and 16 RF chains, respectively. As the values of the LDR noise scalars increase, it is visible that the performance of the HYBF scheme tends closer and closer to the performance of a fully digital HD system. In particular, it is not beneficial to operate in FD mode for LDR noise scalars greater than $-30 \mathrm{~dB}$ at $\mathrm{SNR}=20 \mathrm{~dB}$. Figure 5 shows the average WSR as a function of the LDR noise at $\mathrm{SNR}=40 \mathrm{~dB}$. We can see that the fully digital FD scheme achieves an additional gain of $\sim 82 \%$ compared to a fully digital HD scheme. For $k_{0}=\beta_{0}=\kappa_{k}=\beta_{j} \leq-80 \mathrm{~dB}$, the HYBF scheme achieves around $\sim 65 \%$ and $\sim 56 \%$ additional gain of with 32 and 16 RF chains, respectively, with the unitmodulus phase shifters. With an unconstrained HYBF scheme, the additional gain results to be $\sim 67 \%$ and $61 \%$ with 32 and 


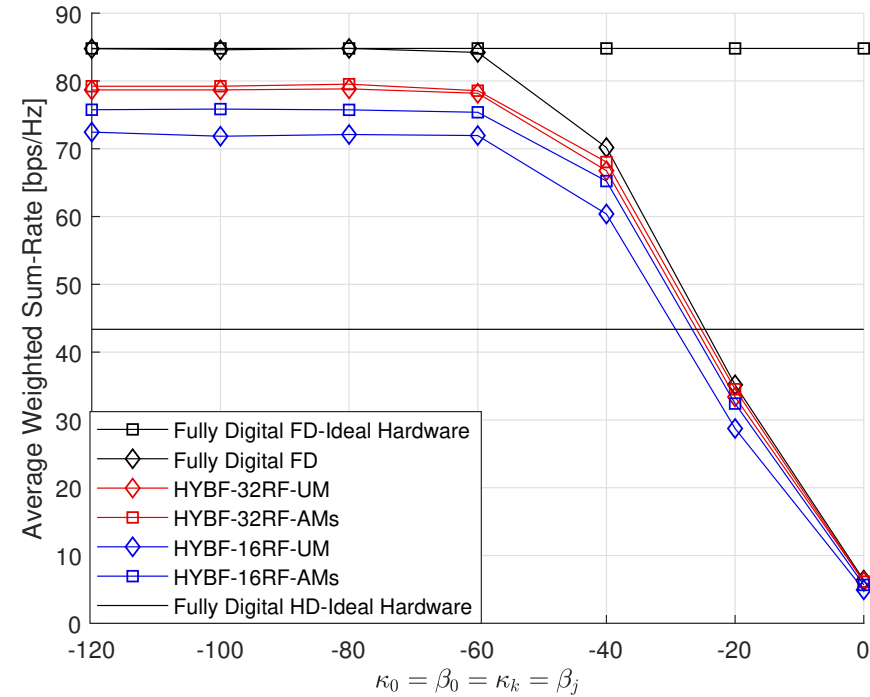

Fig. 4: Average weighted sum rate as a function of the LDR noise with $\mathrm{SNR}=20 \mathrm{~dB}$ with 16 and $32 \mathrm{RF}$ chains.

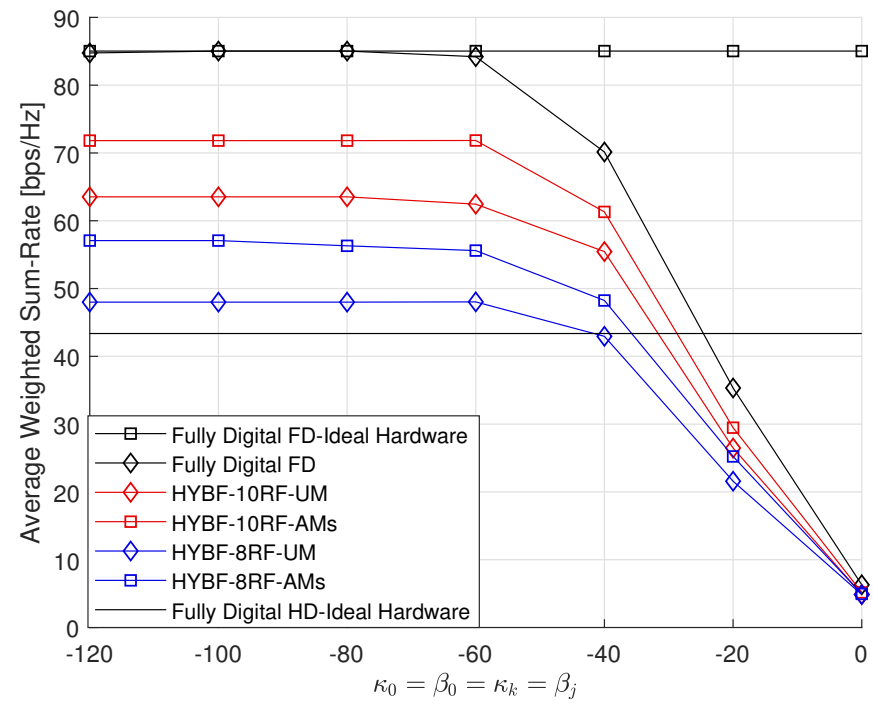

Fig. 6: Average weighted sum rate as a function of the LDR noise with SNR $=20 \mathrm{~dB}$ with 8 and $10 \mathrm{RF}$ chains.

$16 \mathrm{RF}$ chains, respectively. We can also observe that as the SNR increase, the thermal noise level decreases, which leads to the LDR noise dominating the thermal noise floor early, which is observable at $k_{0}=-60 \mathrm{~dB}$, in Figures 4-5.

Figure 6-7 shows the achieved WSR as a function of LDR noise variance at the SNR 20 and $40 \mathrm{~dB}$, respectively, with only 8 and $10 \mathrm{RF}$ chains, both at the transmit and receive side of the hybrid FD base station. Compared to the results reported in Figure 4, in Figure 6 it is visible that the proposed HYBF scheme achieves around $46 \%$ and $11 \%$ additional gain at SNR $=20 \mathrm{~dB}$ with only 10 and $8 \mathrm{RF}$ chains, with the unit-modulus phase shifters for $k_{0}=\beta_{0}=\kappa_{k}=\beta_{j} \leq-60 \mathrm{~dB}$. The AMs provide additional gain of $\sim 19 \%$ and $\sim 20 \%$, leading to an additional gain of $\sim 65 \%$ and $31 \%$ with respect to the fully digital HD system. At the SNR $=40 \mathrm{~dB}$, the gain of a FD system results to be $\sim 40 \%$ and $\sim 14.5 \%$ with 10 and 8 RF

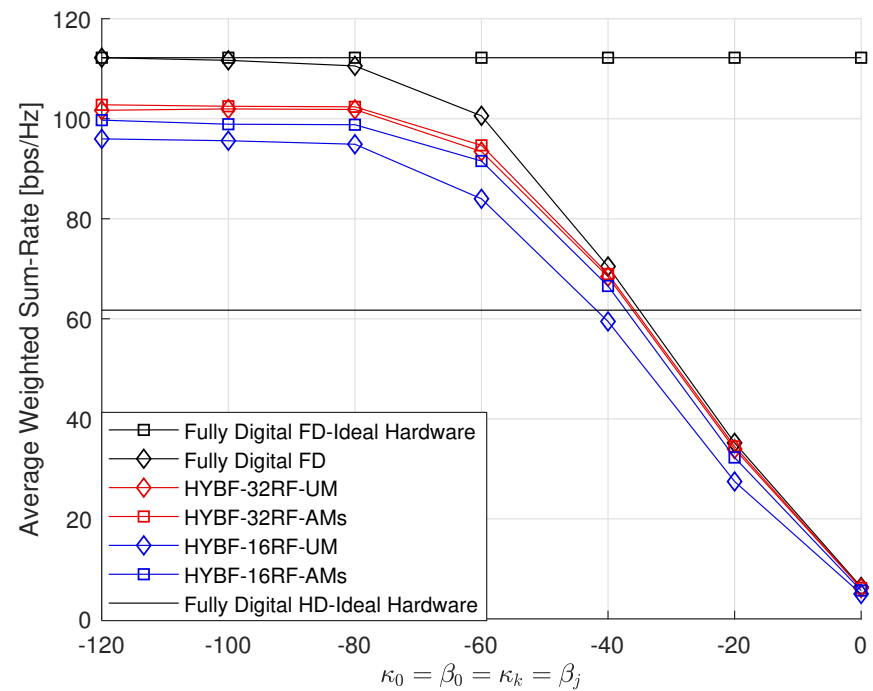

Fig. 5: Average weighted sum rate as a function of the LDR noise with SNR $=40 \mathrm{~dB}$ with 16 and $32 \mathrm{RF}$ chains.

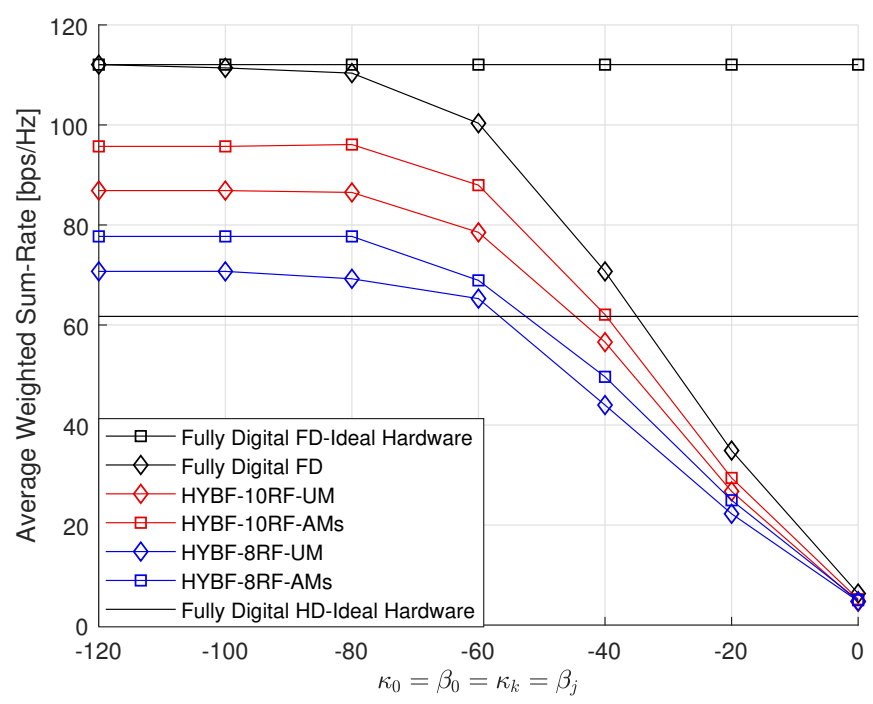

Fig. 7: Average weighted sum rate as a function of the LDR noise with $\mathrm{SNR}=40 \mathrm{~dB}$ with 8 and $10 \mathrm{RF}$ chains.

chains, respectively, with the unit-modulus phase-shifters for $k_{0}=\beta_{0}=\kappa_{k}=\beta_{j} \leq-80 \mathrm{~dB}$. For the unconstrained case, the gain results to be around $\sim 55 \%$ and $\sim 26 \%$ for $k_{0}=\beta_{0}=$ $\kappa_{k}=\beta_{j} \leq-80 \mathrm{~dB}$, with 10 and $8 \mathrm{RF}$ chains, respectively. Therefore, AMs contributes with a performance improvement of $\sim 15 \%$ and $\sim 12 \%$. By comparing the results in Figure 4-5 with results reported in Figure 6-7, we can observe the advantage of having amplitude control at the analog processing stage, when the number of available RF chains is small. In particular, at high SNR $(=40 \mathrm{~dB})$, we can see in Figure 5 and Figure 7 that HYBF with only $10 \mathrm{RF}$ chains and with AMs can perform close to the case of $32 \mathrm{RF}$ chains with unitmodulus phase-shifters, thus saving the hardware cost of $\sim 26$ $\mathrm{RF}$ chains.

Figure 8 shows the performance of the HYBF and combining design in comparison with the benchmark schemes with 


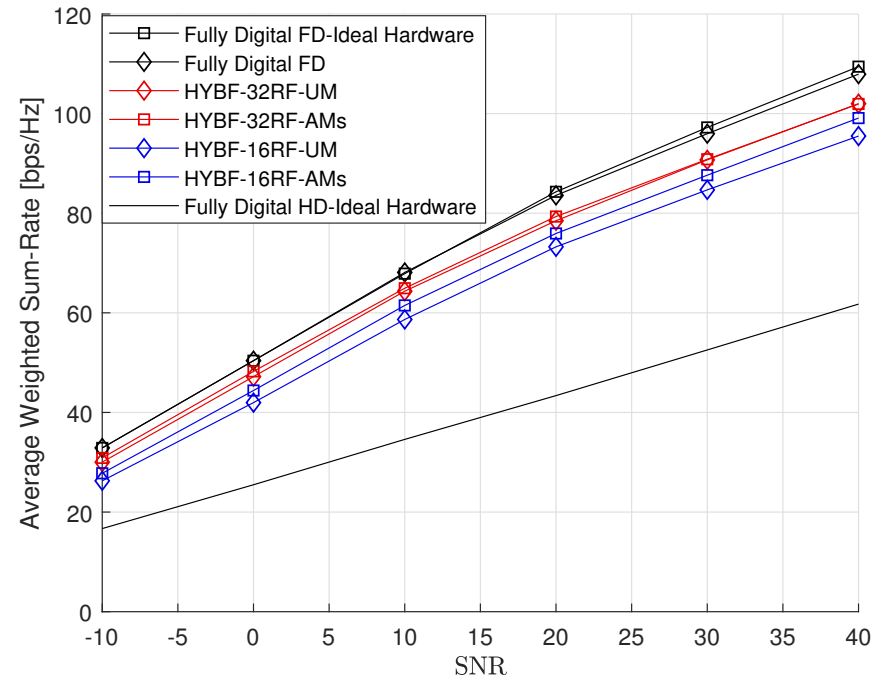

Fig. 8: Average WSR as function of SNR with $k_{0}=\beta_{0}=$ $\kappa_{k}=\beta_{j}=-80 \mathrm{~dB}$, with 16 and $32 \mathrm{RF}$ chains.

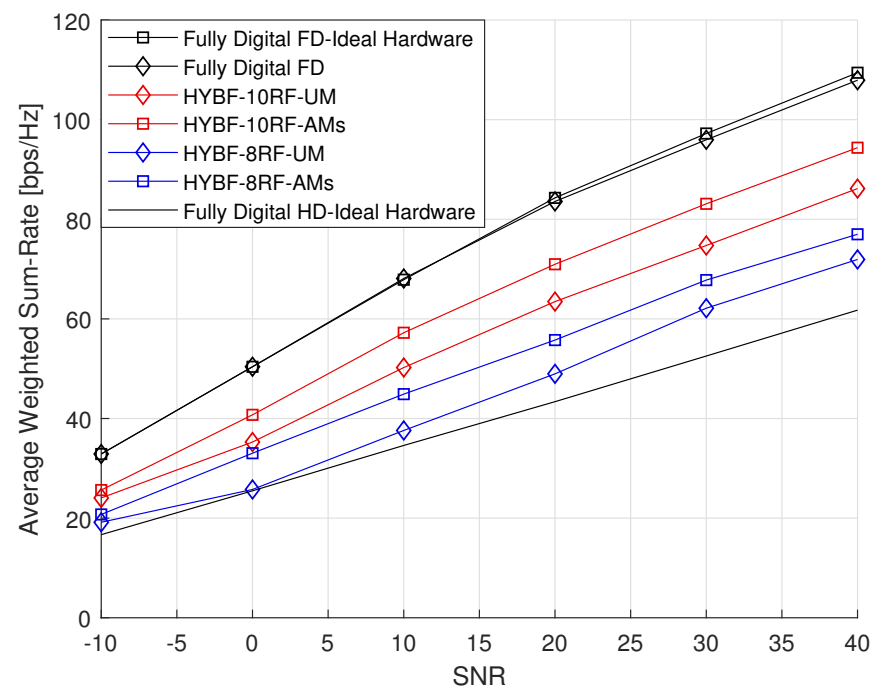

Fig. 10: Average WSR as function of SNR with $k_{0}=\beta_{0}=$ $\kappa_{k}=\beta_{j}=-80 \mathrm{~dB}$, with 8 and $10 \mathrm{RF}$ chains.

$\kappa_{0}=\beta_{0}=\kappa_{k}=\beta_{j}=-80 \mathrm{~dB}$. It is visible that the fully digital FD ideal hardware scheme performs very close to the fully digital FD scheme at low SNR. At SNR $30 \mathrm{~dB}$, the LDR noise variance results to be higher than the thermal noise variance, resulting in slightly less gain for the latter scheme. We can also see that the HYBF UM scheme performs very close to the fully digital FD scheme with $32 \mathrm{RF}$ chains, and using AMs with a high number of RF chains does not bring significant advantage. HYBF UM with 16 RF chains significantly outperforms the fully digital ideal hardware scheme, and the gain $(\sim 8 \%)$ with AMs is much more evident than the $32 \mathrm{RF}$ chains case. Figure 9 shows the WSR as a function of the SNR with $\kappa_{0}=\beta_{0}=\kappa_{k}=\beta_{j}=-60 \mathrm{~dB}$. We can see that for the SNR range $[-10,30] \mathrm{dB}$, the performance of the proposed scheme with a different number of RF chains and with or without AMs results to be similar as in Figure 8. However, at SNR $40 \mathrm{~dB}$, we can see that the WSR tends to

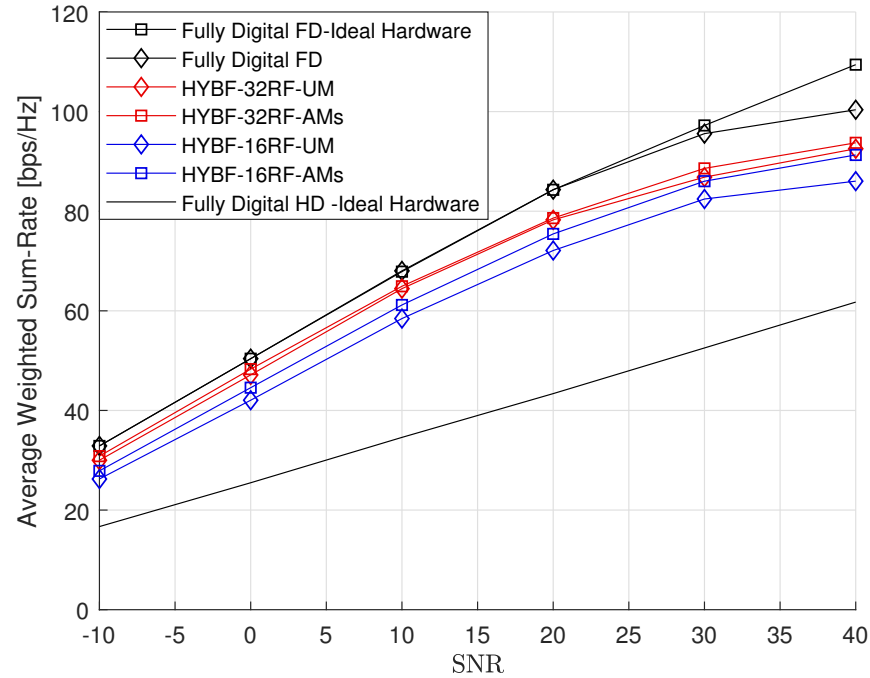

Fig. 9: Average WSR as function of SNR with $k_{0}=\beta_{0}=$ $\kappa_{k}=\beta_{j}=-60 \mathrm{~dB}$, with 16 and $32 \mathrm{RF}$ chains.

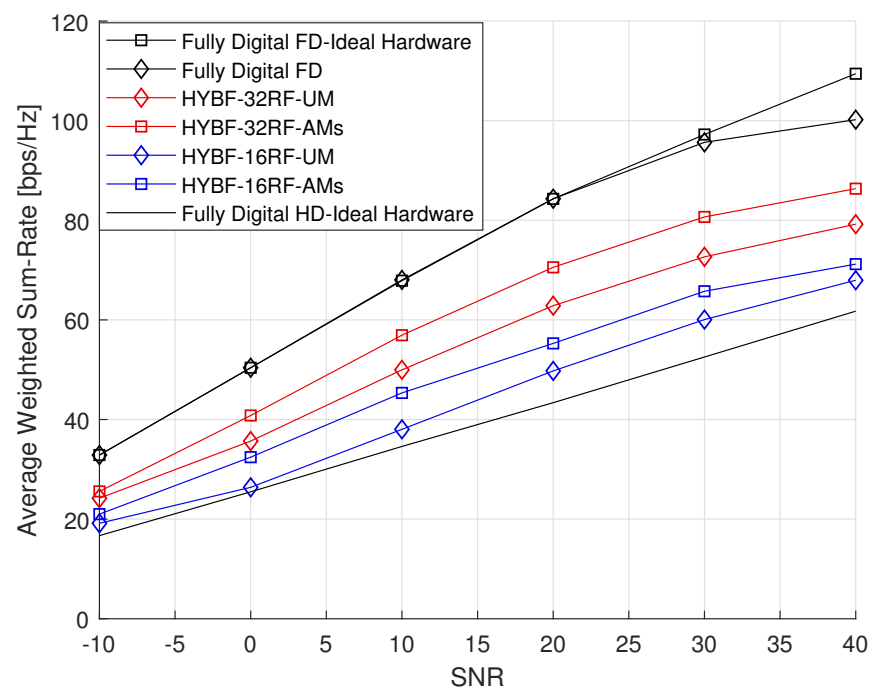

Fig. 11: Average WSR as function of SNR with $k_{0}=\beta_{0}=$ $\kappa_{k}=\beta_{j}=-60 \mathrm{~dB}$, with 8 and $10 \mathrm{RF}$ chains.

suffer significantly. It is also visible some additional marginal gain for the HYBF AMs scheme with $32 \mathrm{RF}$ chains over the HYBF UM scheme when the LDR noise dominates. Figure 10 and Figure 11 shows the WSR as a function of the SNR with $\kappa_{0}=\beta_{0}=\kappa_{k}=\beta_{j}=-80 \mathrm{~dB}$ and $-60 \mathrm{~dB}$, respectively, with 8 and $10 \mathrm{RF}$ chains. We can see that at low SNR with only $8 \mathrm{RF}$ chains, the performance of the HYB UM scheme performs close to the fully digital HD scheme, but AMs bring significant performance improvement. HYB UM scheme with $10 \mathrm{RF}$ chains significantly outperforms the fully digital HD scheme, and equipping it with AMs leads to a significant performance improvement.

Figures 8-11 presented the performance of the HYBF scheme as a function of moderate to low LDR noise variance, under which it is possible to outperform the fully digital HD system with a very few RF chains. Considering a high LDR noise variance, thus having a very low cost circuitry, may 
significantly degrade the performance of a hybrid FD system, which is evident from Figures $4-7$. Having only a very few RF chains (8 or 10) may not bring any additional advantage for an FD system under high LDR noise, as the achieved WSR would be less than the fully digital HD system. To understand the impact of the LDR noise variance and expected WSR, it is crucial to design hybrid FD systems with small values of $k_{0}, \beta_{0}, \kappa_{k}$ and $\beta_{j}$. The values of the LDR noise scalars depend strictly on the RF circuitry present in the FD transceiver. However, these values can be determined with experiments, and the goal of the engineers should be to deploy RF circuitry which introduces the LDR noise variance, such that the LDR noise variance per antenna results proportional to the total transmit or receive power with proportionality factors $\kappa_{0}, \beta_{0}, \kappa_{k}, \beta_{j}<=-60 \mathrm{~dB}$.

From the results presented in Figures 4-11, we can conclude that the proposed HYBF and combining design achieves significant performance improvement in terms of average WSR compared to a fully digital HD system. In particular, Figures 4-5 shows that the LDR noise can result to be a major cause of performance loss for practical hybrid FD transceivers, especially when the number of RF chains is low. Hybrid FD transceivers with a very low LDR range (low-cost circuitry) would require more RF chains to outperform HD systems, which would still increase the hardware cost without benefiting much in terms of performance. Therefore, it is desirable to have a few RF chains with a high dynamic range and AMs, rather than many RF chains with a low dynamic range, which would be beneficial for both the hardware cost and the performance.

\section{CONCLUSION}

This paper has proposed a novel joint HYBF and combining design for WSR maximization in a single-cell mmWave massive MIMO FD systems with multi-antenna HD uplink and downlink users and equipped with non-ideal hardware. The proposed design takes into account the practical per-antenna power constraints, the LDR noise model, the quantized phaseshifters, together with the traditional sum-power constraints. Compared to the state-of-the-art, the results are reported with unit-modulus phase-shifters and unconstrained phase-shifters. Simulation results show significant performance improvement for a mmWave FD system compared to a mmWave HD system. From the results, we can also conclude that the emerging mmWave FD systems with a few RF chains and affected with moderate to low LDR noise can outperform the fully digital HD systems considerably. Moreover, having amplitude control at the analog processing stage can be extremely beneficial when the number of available RF chains is limited.

\section{APPENDIX A \\ GRADIENT DERIVATION}

The ingredients required to prove Theorem 1 are the matrix differentiation calculus and the properties of the trace operator. The proof is based on the results derived in the following.
Lemma 3. Let $\boldsymbol{Y}=\boldsymbol{A} \boldsymbol{X} \boldsymbol{B}+a \boldsymbol{A} \operatorname{diag}(\boldsymbol{X}+\boldsymbol{Q}) \boldsymbol{B}+$ $b \operatorname{diag}(\boldsymbol{C X} \boldsymbol{D}+\boldsymbol{E})+\boldsymbol{F}$, where the size of matrices involved is such that the product is valid. The derivative of $\ln \operatorname{det}(\boldsymbol{Y})$ with respect to $\boldsymbol{X}$ is given by

$$
\begin{aligned}
\frac{\partial \ln \operatorname{det} \boldsymbol{Y}}{\partial \boldsymbol{X}}= & \boldsymbol{A}^{H} \boldsymbol{Y}^{-H} \boldsymbol{B}^{H}+a \operatorname{diag}\left(\boldsymbol{A}^{H} \boldsymbol{Y}^{-H} \boldsymbol{B}^{H}\right) \\
& +b \boldsymbol{C}^{H} \operatorname{diag}\left(\boldsymbol{Y}^{-H}\right) \boldsymbol{D}^{H} .
\end{aligned}
$$

Proof. By substituting $\phi=\ln \operatorname{det}(\boldsymbol{Y})$, we can write

$$
\partial \phi=\boldsymbol{Y}^{-H}: d \boldsymbol{Y}=\operatorname{Tr}\left(\boldsymbol{Y}^{-1} d \boldsymbol{Y}\right)
$$

where the : operator denotes the Frobenius inner product, i.e. $\boldsymbol{G}_{R F}: \boldsymbol{H}=\operatorname{tr}\left(\boldsymbol{G}_{R F}^{T} \boldsymbol{H}\right)$. Its derivative with respect to $\boldsymbol{X}$ can be written as

$$
\begin{aligned}
\frac{\partial \phi}{\partial \boldsymbol{X}} & =\boldsymbol{Y}^{-H}:\left[\frac{d}{\partial \boldsymbol{X}}(\boldsymbol{A} \boldsymbol{X} \boldsymbol{B}+a \boldsymbol{A} \operatorname{diag}(\boldsymbol{X}) \boldsymbol{B}\right. \\
& +b \operatorname{diag}(\boldsymbol{C} \boldsymbol{X} \boldsymbol{D}+\boldsymbol{E})+\boldsymbol{F})],
\end{aligned}
$$

where the last term results to be zero as independent from $\boldsymbol{X}$. Now substituting the Forbenius product with the trace operator and using its cyclic shift property to bring $\boldsymbol{X}$ on the right hand side, we have

$$
\begin{aligned}
& \frac{\partial \phi}{\partial \boldsymbol{X}}=\underbrace{\frac{\partial \operatorname{Tr}\left(\boldsymbol{B} \boldsymbol{Y}^{-1} \boldsymbol{A} \boldsymbol{X}\right)}{\partial \boldsymbol{X}}}_{I}+\underbrace{a \frac{\partial \operatorname{Tr}\left(\boldsymbol{B} \boldsymbol{Y}^{-1} \boldsymbol{A} \operatorname{diag}(\boldsymbol{X})\right)}{\partial \boldsymbol{X}}}_{I I} \\
& +\underbrace{b \frac{\partial \operatorname{Tr}\left(\boldsymbol{Y}^{-1} \operatorname{diag}(\boldsymbol{C} \boldsymbol{X} \boldsymbol{D})\right)}{\partial \boldsymbol{X}}}_{I I I}+b \frac{\partial \operatorname{Tr}\left(\boldsymbol{Y}^{-1} \operatorname{diag}(\boldsymbol{E})\right)}{\partial \boldsymbol{X}},
\end{aligned}
$$

where the last term being independent of $\boldsymbol{X}$ results to be zero. Now we proceed by proving step by step the derivatives for $I, I I$ and $I I I$. Firstly, for $I$ we have

$$
\frac{\partial \operatorname{Tr}\left(\boldsymbol{B} \boldsymbol{Y}^{-1} \boldsymbol{A} \boldsymbol{X}\right)}{\partial \boldsymbol{X}}=\boldsymbol{A}^{H} \boldsymbol{Y}^{-H} \boldsymbol{B}^{H}: d \boldsymbol{X}=\boldsymbol{A}^{H} \boldsymbol{Y}^{-H} \boldsymbol{B}^{H} .
$$

To obtain the derivative of $I I$, let $\operatorname{diag}(\boldsymbol{X})=\boldsymbol{Z}$. A diagonal of $\boldsymbol{X}$ can be written as $\operatorname{diag}(\boldsymbol{X})=\boldsymbol{I} \circ \boldsymbol{X}$ where $\circ$ denote the Hadamard product. By using its commutative property, we can write

$$
\begin{aligned}
a \frac{\partial \operatorname{Tr}\left(\boldsymbol{B} \boldsymbol{Y}^{-1} \boldsymbol{A} \boldsymbol{Z}\right)}{\partial \boldsymbol{Z}} & =a \boldsymbol{A}^{H} \boldsymbol{Y}^{-H} \boldsymbol{B}^{H}: d \boldsymbol{Z}, \\
& =a \boldsymbol{A}^{H} \boldsymbol{Y}^{-H} \boldsymbol{B}^{H}: \boldsymbol{I} \circ d \boldsymbol{X}, \\
& =a \boldsymbol{A}^{H} \boldsymbol{Y}^{-H} \boldsymbol{B}^{H} \circ \boldsymbol{I}: d \boldsymbol{X}, \\
& =a \operatorname{diag}\left(\boldsymbol{A}^{H} \boldsymbol{Y}^{-H} \boldsymbol{B}^{H}\right) .
\end{aligned}
$$

To compute the derivative of $I I I$, let $\operatorname{diag}(\boldsymbol{C} \boldsymbol{X} \boldsymbol{D})=\boldsymbol{W}$ and 
by again using the commutative property of the Hadamard product, we have

$$
\begin{aligned}
b \frac{\partial \operatorname{Tr}\left(\boldsymbol{Y}^{-1} \boldsymbol{W}\right)}{\partial \boldsymbol{W}} & =b \boldsymbol{Y}^{-H}: d \boldsymbol{W}, \\
& =b \boldsymbol{Y}^{-H}: \boldsymbol{I} \circ \boldsymbol{C} d \boldsymbol{X} \boldsymbol{D}, \\
& =b \boldsymbol{Y}^{-H} \circ \boldsymbol{I}: \boldsymbol{C} d \boldsymbol{X} \boldsymbol{D}, \\
& =b \operatorname{diag}\left(\boldsymbol{Y}^{-H}\right): \boldsymbol{C} d \boldsymbol{X} \boldsymbol{D}, \\
& =b \boldsymbol{C}^{H} \operatorname{diag}\left(\boldsymbol{Y}^{-1}\right)^{H} \boldsymbol{D}^{H} .
\end{aligned}
$$

which concludes the proof.

The result provided in Lemma 3 is for the most general case, with any $\boldsymbol{A}, \boldsymbol{B}, \boldsymbol{C}, \boldsymbol{D}, \boldsymbol{E}, \boldsymbol{F}$ and $\boldsymbol{Q}$. To proof the result for Theorem 1, we remark that the covariance matrices has a special (Hermitian) structure, i.e., $\boldsymbol{B}=\boldsymbol{A}^{H}$ and $\boldsymbol{D}=\boldsymbol{C}^{H}$, for any covariance matrix shown in 11 . Therefore, the result of Lemma 3 for this particular is given in the Lemma stated in the following.

Lemma 4. Let $\boldsymbol{Y}=\boldsymbol{A} \boldsymbol{X} \boldsymbol{B}+a \operatorname{Adiag}(\boldsymbol{X}+\boldsymbol{Q}) \boldsymbol{B}+$ $b$ diag $(\boldsymbol{C} \boldsymbol{X} \boldsymbol{D}+\boldsymbol{E})+\boldsymbol{F}$, where the size of matrices involved is such that the product is valid. Let $\boldsymbol{B}=\boldsymbol{A}^{H}$ and $\boldsymbol{D}=\boldsymbol{C}^{H}$ and the derivative of $\ln \operatorname{det}(\boldsymbol{Y})$ is given by

$$
\begin{aligned}
\frac{\partial \ln \operatorname{det} \boldsymbol{Y}}{\partial \boldsymbol{X}}= & \boldsymbol{A}^{H} \boldsymbol{Y}^{-H} \boldsymbol{A}+a \operatorname{diag}\left(\boldsymbol{A}^{H} \boldsymbol{Y}^{-H} \boldsymbol{A}\right) \\
& +b \boldsymbol{C}^{H} \operatorname{diag}\left(\boldsymbol{Y}^{-H}\right) \boldsymbol{C} .
\end{aligned}
$$

Proof. The result follows directly by relying on the result given in Lemma 3 by substituting $\boldsymbol{B}=\boldsymbol{A}^{H}$ and $\boldsymbol{D}=\boldsymbol{C}^{H}$

Proof. Theorem 1 To prove the gradients to linearize the WSR with respect to $\boldsymbol{T}_{k}$ and $\boldsymbol{Q}_{j}$, we proceed by simplifying the WSR as

$$
\begin{aligned}
\mathrm{WSR} & =\sum_{k \in \mathcal{U}} w_{k} \ln \operatorname{det}\left(\boldsymbol{R}_{\bar{k}}^{-1} \boldsymbol{R}_{k}\right)+\sum_{j \in \mathcal{D}} w_{j} \ln \operatorname{det}\left(\boldsymbol{R}_{\bar{j}}^{-1} \boldsymbol{R}_{j}\right) \\
& =\sum_{k \in \mathcal{U}} w_{k}\left(\ln \operatorname{det}\left(\boldsymbol{R}_{k}\right)-\ln \operatorname{det}\left(\boldsymbol{R}_{\bar{k}}\right)\right) \\
& +\sum_{j \in \mathcal{D}} w_{j}\left(\ln \operatorname{det}\left(\boldsymbol{R}_{j}\right)-\ln \operatorname{det}\left(\boldsymbol{R}_{\bar{j}}\right)\right) .
\end{aligned}
$$

The $\mathrm{WSR}_{\bar{k}}^{U L}$ and $\mathrm{WSR}^{D L}$ should be linerized for $\boldsymbol{T}_{k}$ and $\mathrm{WSR}_{j}^{D}{ }^{L}$ and $\mathrm{WSR}^{U L}$ for $\boldsymbol{Q}_{j}$. Note from (11) that $\boldsymbol{T}_{k}$ appears in $\mathrm{WSR}_{\frac{U}{k}}^{L}$ and $\mathrm{WSR}^{D L}$ with the structure $\boldsymbol{Y}=\boldsymbol{A} \boldsymbol{X} \boldsymbol{A}^{H}+$ $a \boldsymbol{A} \operatorname{diag}(\boldsymbol{X}+\boldsymbol{Q}) \boldsymbol{A}^{H}+b \operatorname{diag}\left(\boldsymbol{C} \boldsymbol{X} \boldsymbol{C}^{H}+\boldsymbol{E}\right)+\boldsymbol{F}$, where $a$ and $b$ are due to the LDR noise model, $\boldsymbol{A}$ and $\boldsymbol{C}$ are the interfering channels, $\boldsymbol{F}$ and $\boldsymbol{E}$ contain the noise contributions from other transmit covariance matrices but independent from $\boldsymbol{T}_{k}$. The same structure holds also for the downlink covariance matrices $\boldsymbol{Q}_{j}, \forall j \in \mathcal{D}$. By applying the result from Lemma
4 with $\boldsymbol{Y}=\boldsymbol{R}_{k}$ or $\boldsymbol{Y}=\boldsymbol{R}_{\bar{k}}$ repetitively $K-1$ time for linearizing $\mathrm{WSR}_{\bar{k}}$ with respect to $\boldsymbol{T}_{k}$ yield the gradient $\boldsymbol{A}_{k}$. Similarly, by considering $\boldsymbol{Y}=\boldsymbol{R}_{j}$ or $\boldsymbol{Y}=\boldsymbol{R}_{\bar{j}}, \forall j \in \mathcal{D}$ applying the results from Lemma 4 yield the gradient $\boldsymbol{B}_{k}$.

The same reasoning holds for $\boldsymbol{Q}_{j}$, which leads to the gradients $\hat{\boldsymbol{C}}_{j}$ and $\boldsymbol{D}_{j}$ by applying the result provided in Lemma 4 for $\mathrm{WSR}_{\frac{j}{j}}{ }^{L} J-1$ times and for $\mathrm{WSR}^{U L} K$ times, respectively, $\forall j \in \mathcal{D}$.

\section{APPENDIX B \\ PROOF OF THEOREM 3}

The dominant generalized eigenvector solution maximize the reformulated concave WSR maximization problem

$$
\begin{aligned}
\text { WSR }= & \sum_{k \in \mathcal{U}} w_{k} \ln \operatorname{det}\left(\boldsymbol{I}+\boldsymbol{U}_{k}^{H} \boldsymbol{H}_{k}^{H} \boldsymbol{F}_{R F}^{H} \boldsymbol{R}_{\bar{k}}^{-1} \boldsymbol{F}_{R F} \boldsymbol{H}_{k} \boldsymbol{U}_{k}\right) \\
& -\operatorname{tr}\left(\boldsymbol{U}_{k}^{H}\left(\hat{\boldsymbol{A}}_{k}+\hat{\boldsymbol{B}}_{k}+l_{k} \boldsymbol{I}+\boldsymbol{\Psi}_{k}\right) \boldsymbol{U}_{k}\right) \\
& +\sum_{j \in \mathcal{D}} w_{j} \ln \operatorname{det}\left(\boldsymbol{I}_{d_{j}}+\boldsymbol{V}_{j}^{H} \boldsymbol{G}_{R F}^{H} \boldsymbol{H}_{j}^{H} \boldsymbol{R}_{j}^{-1} \boldsymbol{H}_{j} \boldsymbol{G}_{R F} \boldsymbol{V}_{j}\right) \\
& \left.-\operatorname{tr}\left(\boldsymbol{V}_{j}^{H} \boldsymbol{G}_{R F}^{H}\left(\hat{\boldsymbol{C}}_{j}+\hat{\boldsymbol{D}}_{j}+l_{0} \boldsymbol{I}+\boldsymbol{\Psi}_{0}\right) \boldsymbol{G}_{R F} \boldsymbol{V}_{j}\right)\right) .
\end{aligned}
$$

To prove the results stated in Theorem 3 to solve (49), we first consider the uplink digital beamforming solution by keeping the analog beamformer and the digital downlink beamformers fixed. For the optimal digital uplink beamforming solution, we consider user $k \in \mathcal{U}$. However, the same proof is valid for $\forall k \in \mathcal{U}$. It relies on simplifying

$$
\begin{array}{cc}
\max _{\boldsymbol{U}_{k} .} & w_{k} \ln \operatorname{det}\left(\boldsymbol{I}+\boldsymbol{U}_{k}^{H} \boldsymbol{H}_{k}^{H} \boldsymbol{F}_{R F}^{H} \boldsymbol{R}_{\bar{k}}^{-1} \boldsymbol{F}_{R F} \boldsymbol{H}_{k} \boldsymbol{U}_{k}\right) \\
-\operatorname{tr}\left(\boldsymbol{U}_{k}^{H}\left(\hat{\boldsymbol{A}}_{k}+\hat{\boldsymbol{B}}_{k}+l_{k} \boldsymbol{I}+\boldsymbol{\Psi}_{k}\right) \boldsymbol{U}_{k}\right)
\end{array}
$$

unit the Hadamard's inequality applies as in Proposition 1 [55] or Theorem 1 [58]. The Cholesky decomposition of the matrix $\left(\hat{\boldsymbol{A}}_{k}+\hat{\boldsymbol{B}}_{k}+l_{k}+\boldsymbol{\Psi}_{k}\right)$ is written as $\boldsymbol{L}_{k} \boldsymbol{L}_{k}^{H}$ where $\boldsymbol{L}_{k}$ is the lower triangular Cholesky factor. By defining $\tilde{\boldsymbol{U}}_{k}=\boldsymbol{L}_{k}^{H} \boldsymbol{U}_{k}$, (50) reduces to

$$
\begin{aligned}
\max _{\boldsymbol{U}_{k}} . w_{k} \ln \operatorname{det}\left(\boldsymbol{I}+\tilde{\boldsymbol{U}}_{k}^{H} \boldsymbol{L}_{k}^{-1} \boldsymbol{H}_{k}^{H} \boldsymbol{F}_{R F}^{H} \boldsymbol{R}_{\bar{k}}^{-1} \boldsymbol{F}_{R F} \boldsymbol{H}_{k} \boldsymbol{L}_{k}^{-H} \tilde{\boldsymbol{U}}_{k}\right) \\
-\operatorname{tr}\left(\tilde{\boldsymbol{U}}_{k}{ }^{H} \tilde{\boldsymbol{U}}_{k}\right) .
\end{aligned}
$$

Let $\boldsymbol{E}_{k} \boldsymbol{D}_{k} \boldsymbol{E}_{k}^{H}$ be the eigen-decomposition of $\boldsymbol{L}_{k}^{-1} \boldsymbol{H}_{k}^{H} \boldsymbol{R}_{\bar{k}}^{-1} \boldsymbol{H}_{k} \boldsymbol{L}_{k}^{-H}$, where $\boldsymbol{E}_{k}$ and $\boldsymbol{D}_{k}$ are the unitary and diagonal matrices, respectively. Let $\boldsymbol{O}_{k}=\boldsymbol{E}_{k}^{H} \tilde{\boldsymbol{U}}_{k} \tilde{\boldsymbol{U}}_{k}{ }^{H} \boldsymbol{E}_{k}$, the (51) can be expressed as

$$
\max _{\boldsymbol{O}_{k}} \quad w_{k} \ln \operatorname{det}\left(\boldsymbol{I}_{u_{k}}+\boldsymbol{O}_{k} \boldsymbol{D}_{k}\right)-\operatorname{tr}\left(\boldsymbol{O}_{k}\right) .
$$

By Hadamard's inequality [Page 233 [59]], it can be seen that the optimal $\boldsymbol{O}_{k}$ must be diagonal. Therefore, $\boldsymbol{U}_{k}=$ 
$\boldsymbol{L}_{k}^{-H} \boldsymbol{E}_{k} \boldsymbol{O}_{k}^{\frac{1}{2}}$ and thus

$$
\begin{array}{r}
\boldsymbol{H}_{k}^{H} \boldsymbol{F}_{R F}^{H} \boldsymbol{R}_{\bar{k}}^{-1} \boldsymbol{F}_{R F} \boldsymbol{H}_{k} \boldsymbol{U}_{k}=\boldsymbol{L}_{k} \boldsymbol{L}_{k}^{H} \boldsymbol{L}_{k}^{-H} \boldsymbol{E}_{k} \boldsymbol{O}_{k}^{\frac{1}{2}} \boldsymbol{D}_{k} \\
=\left(\hat{\boldsymbol{A}}_{k}+\hat{\boldsymbol{B}}_{k}+l_{k}+\boldsymbol{\Psi}_{k}\right) \boldsymbol{U}_{k} \boldsymbol{D}_{k},
\end{array}
$$

from which we select $u_{k}$ dominant eigenvectors and it concludes the proof for the uplink beamformer for user $k \in \mathcal{U}$. For the digital downlink beamformers, the aforementioned result holds immediately by applying on the optimization problem

$$
\begin{aligned}
\max _{\boldsymbol{V}_{j}} & \sum_{j \in \mathcal{D}} w_{j} \ln \operatorname{det}\left(\boldsymbol{I}_{d_{j}}+\boldsymbol{V}_{j}^{H} \boldsymbol{G}_{R F}^{H} \boldsymbol{H}_{j}^{H} \boldsymbol{R}_{\bar{j}}^{-1} \boldsymbol{H}_{j} \boldsymbol{G}_{R F} \boldsymbol{V}_{j}\right) \\
& \left.-\operatorname{tr}\left(\boldsymbol{V}_{j}^{H} \boldsymbol{G}_{R F}^{H}\left(\hat{\boldsymbol{C}}_{j}+\hat{\boldsymbol{D}}_{j}+l_{0}+\boldsymbol{\Psi}_{0}\right) \boldsymbol{G}_{R F} \boldsymbol{V}_{j}\right)\right) .
\end{aligned}
$$

and simplifying it until the Hadamard's inequality applies to yield a result as expressed in (53).

The proof for the analog beamformer does not apply directly as the KKT conditions have the form $\boldsymbol{A}_{1} \boldsymbol{G}_{R F} \boldsymbol{A}_{2}=$ $\boldsymbol{B}_{1} \boldsymbol{G}_{R F} \boldsymbol{B}_{2}$. To solve it for the analog beamformer $\boldsymbol{G}_{R F}$, we apply the result $\operatorname{vec}(\boldsymbol{A} \boldsymbol{X} \boldsymbol{B})=\boldsymbol{B}^{T} \otimes \boldsymbol{A v e c}(\boldsymbol{X})$ [60], which allows to rewrite (23) as

$$
\begin{aligned}
\left(\left(\boldsymbol{V}_{j} \boldsymbol{V}_{j}^{H}\left(\boldsymbol{I}+\boldsymbol{V}_{j} \boldsymbol{V}_{j}^{H} \boldsymbol{G}_{R F}^{H} \boldsymbol{H}_{j}^{H} \boldsymbol{R}_{j}^{-1} \boldsymbol{H}_{j} \boldsymbol{G}_{R F}\right)^{-1}\right)^{T} \otimes\right. \\
\left.\boldsymbol{H}_{j}^{H} \boldsymbol{R}_{\bar{j}}^{-1} \boldsymbol{H}_{j}\right) \operatorname{vec}\left(\boldsymbol{G}_{R F}\right)-\left(( \boldsymbol { V } _ { j } \boldsymbol { V } _ { j } ^ { H } ) ^ { T } \otimes \left(\hat{\boldsymbol{C}}_{j}+\hat{\boldsymbol{D}}_{j}\right.\right. \\
\left.\left.+\boldsymbol{\Psi}_{0}+l_{0} \boldsymbol{I}\right)\right) \operatorname{vec}\left(\boldsymbol{G}_{R F}\right)=0 .
\end{aligned}
$$

The analog beamformer solution can alternatively be derived as follows (which allows the proof above to be applicable directly). First we apply a noise whitening procedure using the noise plus interference covariance matrix $\mathbf{R}_{j}^{1 / 2}$ on the received signal. Further, we can rewrite the whitened signal as follows

$$
\widetilde{\boldsymbol{y}}_{j}=\left(\left(\boldsymbol{s}_{j_{d}}^{T} \boldsymbol{V}_{j}^{T}\right) \otimes \mathbf{R}_{\bar{j}}^{-1 / 2} \boldsymbol{H}_{j}\right) \operatorname{vec}\left(\boldsymbol{G}_{R F}\right)+\widetilde{\mathbf{n}}_{j},
$$

where $\widetilde{\mathbf{y}}_{j}=\mathbf{R}_{\bar{j}}^{-1 / 2} \mathbf{y}_{j}$ and $\widetilde{\mathbf{n}}_{j}$ represents the whitened noise plus interference signal. Further, we can write the resulting WSR optimization problem (after the approximation to concave form and some algebraic manipulations on the linearized term) as

$$
\begin{gathered}
\max _{\boldsymbol{G}_{R F}} \sum_{j \in \mathcal{D}} w_{j} \ln \operatorname{det}\left(\boldsymbol{I}+\operatorname{vec}\left(\boldsymbol{G}_{R F}\right)^{H}\left(\left(\boldsymbol{V}_{j} \boldsymbol{V}_{j}^{H}\right)^{T} \otimes \boldsymbol{H}_{j}^{H} \boldsymbol{R}_{j}^{-1}\right.\right. \\
\left.\left.\boldsymbol{H}_{j}\right) \operatorname{vec}\left(\boldsymbol{G}_{R F}\right)\right)-\operatorname{Tr}\left(\operatorname { v e c } ( \boldsymbol { G } _ { R F } ) ^ { H } \left(\boldsymbol{V}_{j} \boldsymbol{V}_{j}^{H} \otimes\right.\right. \\
\left.\left.\left(\hat{\boldsymbol{C}}_{j}+\hat{\boldsymbol{D}}_{j}\right)+\boldsymbol{\Psi}_{0}+l_{0} \boldsymbol{I}\right) \operatorname{vec}\left(\boldsymbol{G}_{R F}\right)\right) .
\end{gathered}
$$

Further, taking the gradient for $\mathbf{G}_{R F}$ leads to the same generalized eigenvector solution as in (24). Also, note that this alternative representation has the same form as (50). Hence, the proof for the uplink and downlink digital beamformers can now be applied directly on the vectorized analog beamformer $\operatorname{vec}\left(\boldsymbol{G}_{R F}\right)$.

\section{ACKNOWLEDGMENT}

The research leading to these results received funding from the French National Research Agency (ANR) via the DUPLEX project. EURECOMs research is also partially supported by its industrial members: ORANGE, BMW, Symantec, SAP, Monaco Telecom, iABG, by the projects MASS-START (French FUI) and EU ITN project SPOTLIGHT.

\section{REFERENCES}

[1] Z. Pi and F. Khan, "An introduction to millimeter-wave mobile broadband systems," IEEE communications magazine, vol. 49, no. 6, pp. 101107, 2011.

[2] S. Rangan, T. S. Rappaport, and E. Erkip, "Millimeter-wave cellular wireless networks: Potentials and challenges," Proceedings of the IEEE, vol. 102, no. 3, pp. 366-385, 2014.

[3] S. Liu, L. Fu, and W. Xie, "Hidden-node problem in full-duplex enabled csma networks," IEEE Transactions on Mobile Computing, vol. 19, no. 2, pp. 347-361, 2019.

[4] K. Pärlin and T. Riihonen, "Full-duplex transceivers for defense and security applications," in Full-Duplex Communications for Future Wireless Networks. Springer, 2020, pp. 249-274.

[5] M. T. Kabir and C. Masouros, "A scalable energy vs. latency trade-off in full-duplex mobile edge computing systems," IEEE Transactions on Communications, vol. 67, no. 8, pp. 5848-5861, 2019.

[6] C. B. Barneto, S. D. Liyanaarachchi, M. Heino, T. Riihonen, and M. Valkama, "Full duplex radio/radar technology: The enabler for advanced joint communication and sensing," IEEE Wireless Communications, vol. 28, no. 1, pp. 82-88, 2021.

[7] M. Gan, Y. Guo, G. Tsodik, Y. Xin, X. Yang, E. Au, and O. AboulMagd, "Full duplex for next generation of 802.11," in 2019 IEEE 30th International Symposium on Personal, Indoor and Mobile Radio Communications (PIMRC Workshops). IEEE, 2019, pp. 1-6.

[8] P. Rosson, C. K. Sheemar, N. Valecha, and D. Slock, "Towards massive mimo in-band full duplex radio," in 2019 16th International Symposium on Wireless Communication Systems (ISWCS). IEEE, 2019, pp. 69-74.

[9] S. Huberman and T. Le-Ngoc, "Mimo full-duplex precoding: A joint beamforming and self-interference cancellation structure," IEEE Transactions on Wireless Communications, vol. 14, no. 4, pp. 2205-2217, 2014.

[10] P. Aquilina, A. C. Cirik, and T. Ratnarajah, "Weighted sum rate maximization in full-duplex multi-user multi-cell mimo networks," IEEE Transactions on Communications, vol. 65, no. 4, pp. 1590-1608, 2017.

[11] T. Riihonen and R. Wichman, "Analog and digital self-interference cancellation in full-duplex mimo-ofdm transceivers with limited resolution in a/d conversion," in 2012 conference record of the forty sixth asilomar conference on signals, systems and computers (ASILOMAR). IEEE, 2012, pp. 45-49.

[12] B. P. Day, A. R. Margetts, D. W. Bliss, and P. Schniter, "Full-duplex bidirectional mimo: Achievable rates under limited dynamic range," IEEE Transactions on Signal Processing, vol. 60, no. 7, pp. 3702-3713, 2012.

[13] O. Taghizadeh, J. Zhang, and M. Haardt, "Transmit beamforming aided amplify-and-forward mimo full-duplex relaying with limited dynamic range," Signal Processing, vol. 127, pp. 266-281, 2016.

[14] A. C. Cirik, S. Biswas, S. Vuppala, and T. Ratnarajah, "Beamforming design for full-duplex mimo interference channels-qos and energyefficiency considerations," IEEE Transactions on Communications, vol. 64, no. 11, pp. 4635-4651, 2016.

[15] E. Antonio-Rodríguez, R. López-Valcarce, T. Riihonen, S. Werner, and R. Wichman, "Sinr optimization in wideband full-duplex mimo relays under limited dynamic range," in 2014 IEEE 8th Sensor Array and Multichannel Signal Processing Workshop (SAM). IEEE, 2014, pp. 177-180.

[16] S. Biswas, K. Singh, O. Taghizadeh, and T. Ratnarajah, "Design and analysis of fd mimo cellular systems in coexistence with mimo radar," IEEE Transactions on Wireless Communications, vol. 19, no. 7, pp. 4727-4743, 2020. 
[17] A. C. Cirik, O. Taghizadeh, L. Lampe, R. Mathar, and Y. Hua, "Linear transceiver design for full-duplex multi-cell mimo systems," IEEE Access, vol. 4, pp. 4678-4689, 2016.

[18] O. Taghizadeh, V. Radhakrishnan, A. C. Cirik, R. Mathar, and L. Lampe, "Hardware impairments aware transceiver design for bidirectional fullduplex mimo ofdm systems," IEEE Transactions on Vehicular Technology, vol. 67, no. 8, pp. 7450-7464, 2018.

[19] K. Satyanarayana, M. El-Hajjar, P.-H. Kuo, A. Mourad, and L. Hanzo, "Hybrid beamforming design for full-duplex millimeter wave communication," IEEE Transactions on Vehicular Technology, vol. 68, no. 2 , pp. 1394-1404, 2018.

[20] H. Abbas and K. Hamdi, "Full duplex relay in millimeter wave backhaul links," in 2016 IEEE Wireless Communications and Networking Conference. IEEE, 2016, pp. 1-6.

[21] R. López-Valcarce and N. González-Prelcic, "Analog beamforming for full-duplex millimeter wave communication," in 2019 16th International Symposium on Wireless Communication Systems (ISWCS). IEEE, 2019, pp. 687-691.

[22] S. Han, Y. Zhang, W. Meng, C. Li, and Z. Zhang, "Full-duplex relayassisted macrocell with millimeter wave backhauls: Framework and prospects," IEEE Network, vol. 33, no. 5, pp. 190-197, 2019.

[23] C. K. Sheemar and D. T. Slock, "Hybrid beamforming for bidirectional massive mimo full duplex under practical considerations," in VTC Spring 2021, IEEE Vehicular Technology Conference, 25-28 April 2021, Helsinki, Finland, IEEE, Ed., Helsinki, 2021.

[24] Y. Cai, K. Xu, A. Liu, M. Zhao, B. Champagne, and L. Hanzo, "Twotimescale hybrid analog-digital beamforming for mmwave full-duplex mimo multiple-relay aided systems," IEEE Journal on Selected Areas in Communications, 2020.

[25] S. Huang, Y. Ye, and M. Xiao, "Learning based hybrid beamforming design for full-duplex millimeter wave systems," arXiv preprint arXiv:2004.08285, 2020.

[26] C. K. Thomas, C. K. Sheemar, and D. Slock, "Multi-stage/hybrid bf under limited dynamic range for ofdm fd backhaul with mimo si nulling," in 2019 16th International Symposium on Wireless Communication Systems (ISWCS). IEEE, 2019, pp. 96-101.

[27] E. Balti, N. Mensi, and S. Yan, "A modified zero-forcing max-power design for hybrid beamforming full-duplex systems," arXiv preprint arXiv:2003.00147, 2020.

[28] M.-M. Zhao, Y. Cai, M.-J. Zhao, Y. Xu, and L. Hanzo, "Robust joint hybrid analog-digital transceiver design for full-duplex mmwave multicell systems," IEEE Transactions on Communications, 2020.

[29] J. M. B. da Silva, A. Sabharwal, G. Fodor, and C. Fischione, "1-bit phase shifters for large-antenna full-duplex mmwave communications," IEEE Transactions on Wireless Communications, vol. 19, no. 10, pp 6916-6931, 2020

[30] I. P. Roberts, J. G. Andrews, and S. Vishwanath, "Hybrid beamforming for millimeter wave full-duplex under limited receive dynamic range," arXiv preprint arXiv:2012.11647, 2020.

[31] C. K. Sheemar and D. Slock, "Massive mimo mmwave full duplex relay for iab with limited dynamic range," in 2021 11th IFIP International Conference on New Technologies, Mobility and Security (NTMS). IEEE, 2021, pp. 1-5.

[32] J. Palacios, J. Rodriguez-Fernandez, and N. González-Prelcic, "Hybrid precoding and combining for full-duplex millimeter wave communication," in 2019 IEEE Global Communications Conference (GLOBECOM). IEEE, 2019, pp. 1-6.

[33] I. P. Roberts and S. Vishwanath, "Beamforming cancellation design for millimeter-wave full-duplex," in 2019 IEEE Global Communications Conference (GLOBECOM). IEEE, 2019, pp. 1-6.

[34] I. P. Roberts, H. B. Jain, and S. Vishwanath, "Equipping millimeterwave full-duplex with analog self-interference cancellation," in 2020 IEEE International Conference on Communications Workshops (ICC Workshops). IEEE, 2020, pp. 1-6.

[35] C. K. Sheemar and D. Slock, "Hybrid beamforming and combining for millimeter wave full duplex massive mimo interference channel," arXiv preprint arXiv:2108.00465, 2021.

[36] I. P. Roberts, H. B. Jain, and S. Vishwanath, "Frequency-selective beamforming cancellation design for millimeter-wave full-duplex," in ICC 2020-2020 IEEE International Conference on Communications (ICC). IEEE, 2020, pp. 1-6.

[37] D. Korpi, T. Riihonen, V. Syrjälä, L. Anttila, M. Valkama, and R. Wichman, "Full-duplex transceiver system calculations: Analysis of adc and linearity challenges," IEEE Transactions on Wireless Communications, vol. 13, no. 7, pp. 3821-3836, 2014.
[38] C. K. Sheemar, L. Badia, and S. Tomasin, "Game-theoretic mode scheduling for dynamic tdd in 5g systems," IEEE Communications Letters, 2021.

[39] J. M. B. da Silva, G. Wikström, R. K. Mungara, and C. Fischione, "Full duplex and dynamic tdd: Pushing the limits of spectrum reuse in multicell communications," IEEE Wireless Communications, vol. 28, no. 1, pp. 44-50, 2021.

[40] H. Kim, J. Kim, and D. Hong, "Dynamic tdd systems for $5 \mathrm{~g}$ and beyond: A survey of cross-link interference mitigation," IEEE Communications Surveys \& Tutorials, vol. 22, no. 4, pp. 2315-2348, 2020.

[41] E. de Olivindo Cavalcante, G. Fodor, Y. C. Silva, and W. C. Freitas, "Bidirectional sum-power minimization beamforming in dynamic tdd mimo networks," IEEE Transactions on Vehicular Technology, vol. 68 , no. 10 , pp. 9988-10002, 2019.

[42] S. Guo, X. Hou, and H. Wang, "Dynamic tdd and interference management towards 5g," in 2018 IEEE Wireless Communications and Networking Conference (WCNC). IEEE, 2018, pp. 1-6.

[43] E. Björnson, L. Sanguinetti, and M. Kountouris, "Deploying dense networks for maximal energy efficiency: Small cells meet massive mimo," IEEE Journal on Selected Areas in Communications, vol. 34, no. 4, pp. 832-847, 2016.

[44] M. R. Castellanos, V. Raghavan, J. H. Ryu, O. H. Koymen, J. Li, D. J. Love, and B. Peleato, "Hybrid multi-user precoding with amplitude and phase control," in 2018 IEEE International Conference on Communications (ICC). IEEE, 2018, pp. 1-6.

[45] M. Majidzadeh, J. Kaleva, N. Tervo, H. Pennanen, A. Tölli, and M. Latva-Aho, "Rate maximization for partially connected hybrid beamforming in single-user mimo systems," in 2018 IEEE 19th International Workshop on Signal Processing Advances in Wireless Communications (SPAWC). IEEE, 2018, pp. 1-5.

[46] P. Stoica and Y. Selén, "Cyclic Minimizers, Majorization Techniques, and the Expectation-Maximization Algorithm: A Refresher," IEEE Signal Processing Magazine, Jan. 2004.

[47] W. Yu and T. Lan, "Transmitter optimization for the multi-antenna downlink with per-antenna power constraints," IEEE Transactions on signal processing, vol. 55, no. 6, pp. 2646-2660, 2007.

[48] T. Lan and W. Yu, "Input optimization for multi-antenna broadcast channels with per-antenna power constraints," in IEEE Global Telecommunications Conference, 2004. GLOBECOM'04., vol. 1. IEEE, 2004, pp. 420-424.

[49] R. Chaluvadi, S. S. Nair, and S. Bhashyam, "Optimal multi-antenna transmission with multiple power constraints," IEEE Transactions on Wireless Communications, vol. 18, no. 7, pp. 3382-3394, 2019.

[50] C. K. Sheemar and D. T. Slock, "Beamforming for bidirectional mimo full duplex under the joint sum power and per antenna power constraints," in ICASSP 2021, IEEE International Conference on Acoustics, Speech and Signal Processing, 6-11 June 2021, Toronto, Canada, IEEE, Ed., Toronto, 2021.

[51] E. G. Larsson, O. Edfors, F. Tufvesson, and T. L. Marzetta, "Massive mimo for next generation wireless systems," IEEE communications magazine, vol. 52, no. 2, pp. 186-195, 2014.

[52] L. Chen, A. Liu, and X. Yuan, "Structured turbo compressed sensing for massive mimo channel estimation using a markov prior," IEEE Transactions on Vehicular Technology, vol. 67, no. 5, pp. 4635-4639, 2017.

[53] I. P. Roberts, J. G. Andrews, H. B. Jain, and S. Vishwanath, "Millimeterwave full duplex radios: New challenges and techniques," IEEE Wireless Communications, vol. 28, no. 1, pp. 36-43, 2021.

[54] S. S. Christensen, R. Agarwal, E. De Carvalho, and J. M. Cioffi, "Weighted sum-rate maximization using weighted mmse for mimo-bc beamforming design," IEEE Transactions on Wireless Communications, vol. 7, no. 12, pp. 4792-4799, 2008.

[55] S.-J. Kim and G. B. Giannakis, "Optimal resource allocation for mimo ad hoc cognitive radio networks," IEEE Transactions on Information Theory, vol. 57, no. 5, pp. 3117-3131, 2011.

[56] S. Boyd, S. P. Boyd, and L. Vandenberghe, Convex optimization. Cambridge university press, 2004.

[57] S. Boyd and L. Vandenberghe, "Convex optimization," in Cambridge, U.K.: Cambridge Univ. Press, 2004.

[58] D. Hoang and R. A. Iltis, "Noncooperative eigencoding for mimo ad hoc networks," IEEE Transactions on Signal Processing, vol. 56, no. 2 , pp. 865-869, 2008.

[59] T. M. Cover, Elements of information theory. John Wiley \& Sons, 1999.

[60] J. R. Magnus and H. Neudecker, Matrix differential calculus with applications in statistics and econometrics. John Wiley \& Sons, 2019. 